\title{
CHELAT- UND NICHT-CHELAT-KONTROLLIERTE REDUKTIONEN VON B-AMIDO-KETONEN SYNTHESE NICHT-RACEMISCHER 1,3-AMINOALKOHOLE MIT PYRROLIDINSTRUKTUR
}

\author{
KLAUS TH WANNER** und GEORG HÖFNER \\ Institut für Pharmazie und Lebensmittelchemie der Universitat Munchen \\ Sophienstr 10,8000 Munchen 2, BRD \\ "Neue Anschrift Institut für Pharmazie der Freien Unıversităt Berlın \\ Könıgin-Luıse-Str 2+4, 1000 Berlın 33, BRD
}

(Recelved in Germany 25 October 1990)

\begin{abstract}
The reduction of the amidoketones $(R)-1$ and $(S)-2$ has been achieved in a stereocontrolled manner giving either the product with like- or unlike configuration depending upon the reducing agent employed Thus with $\mathrm{L} 1\left(\mathrm{O}^{t} \mathrm{Bu}\right)_{3} \mathrm{AlH}$ the amidoalcohols $(R, R)-3$ and $(S, S)-5$ were the major diastereomers formed whereas with $\mathrm{K}\left({ }^{\mathrm{s}} \mathrm{Bu}\right)_{3} \mathrm{BH}(R, S)-4$ and $(S, R)-6$ dominated. Stereoselectuvities up to $98 / 2$ were observed As a mechanistic rationale for the change of direction in the asymmetric induction a chelate- and non-chelate-model have been envoked

By including ( $\mathrm{rac}$ )-9 in this study having a non chiral acyl group it was found that the chiral auxiliary in $(R)-1 /(S)-2$ is essential for a high asymmetric induction under chelate- but not under non-chelate-control.

Hydrolysis of the amidoalcohols provided the corresponding 1,3-aminoalcohols $(R, R)-12,(S, S)-12,(R, S)-13$ and $(S, R)-13$. The configuration of these compounds has been assigned by means of ${ }^{1} \mathrm{H}$ NMR spectroscopy
\end{abstract}

1,3-Amınoalkohole sind in der Natur wert verbreitet So tritt dieses Strukturelement $\mathbf{u}$ a bel Amınosäuren ${ }^{1}$ und Alkaloıden ${ }^{2}$ auf, als Beispiele seien hier (-)-Hygrolın, (+)-Pseudohygrolın, (+)-Darlıngianın, (-)-Sedamın und (-)-Allosedamin genannt In dieser Substanzklasse sind auch viele pharmakologisch aktıve Verbindungen synthetsschen Ursprungs, wie z B 2-Pyrrolidinyl- ${ }^{3}$ und 1-Tetrahydroisochinolylethanole, ${ }^{4}$ anzutreffen Fur die Darstellung von 1,3-Amınoalkoholen 1st mittlerweile ein breites Arsenal praparatıver Methoden verfugbar So kann man, zB, 1,3-Amınoalkohole durch Cyclisierung von $\mathrm{N}-1 \mathrm{a}, 5$ bzw O-Homoallylcarbamaten ${ }^{6}$ erhalten

Ein anderer, flexıbler Zugang wurde mit der reduktıven Rıngöffnung von Isoxazolınen ${ }^{7}$ und Isoxazolıdınen ${ }^{8}$ geschaffen, welche durch Cycloadditionsreaktionen von Olefinen mit Nitriloxiden bzw Nitronen darstellbar sind Eine naheliegende und vielfach genutzte Lösung besteht schließlıch dann, B-Hydroxyımıne bzw -oxıme ${ }^{9}$ oder B-Aminoketone und ihre Denvate ${ }^{10}$ für die 1,3-Aminoalkoholdarstellung heranzuziehen

Die Mehrzahl dieser Verfahren sind auch als asymmetrische Synthese durchfuhrbar - mit chiralen Hulfsgruppen, oder als Chiral-Pool-Synthesen Der direkte Weg uber die B-Aminoketone ist dafur jedoch wenig geeıgnet, soll die Induktıon vom $\alpha$-C-Atom zum Stıckstoff ausgehen, da Chiralitätszentren an dieser Stelle bei B-Aminoketonen nicht konfigurationsstabil ${ }^{11}$ sind. Anders bei N-Acyl- und N-Alkoxycarbonyl-B-aminoketonen, fur deren Darstellung in optusch aktiver Form sind mittlerweile auch einige Verfahren ${ }^{12}$ verfügbar

Ein neues, besonders einfaches, das wir als "Asymmetrische Elektrophile $\alpha$-Amidoalkylierung" (AE $\alpha$ A) 
bezeichnen, besteht nun dann, $\mathrm{N}$-Acyliminumionen mit einem chiralen $\mathrm{N}$-Acylrest als Hulfsgruppe stereoselektıv mit "Keton-Nukleophilen" abzufangen, um so das Chiralıtätszentrum am $\alpha$-C-Atom zum Amidsuckstoff aufzubauen. ${ }^{13}$ In Fortsetzung unserer Arbeiten zur Darstellung optusch aktnver 1,3-Aminoalkohole haben wir jetzt untersucht, unter welchen Bedingungen sich die so zu erhaltenden Amidoketone stereoselektıv zu Amidoalkoholen reduzieren lassen Mit den Reduktionsversuchen wurde an den Verbindungen ${ }^{14}(R)-1 /(S)-2$ begonnen.

\section{$\underline{\text { Reduktionsversuche }}$}

Die Reduktionen der Amidoketone ( $R$ )-1a-e zu den Amidoalkoholen $(R, R)-3 /(R, S)-4 \mathrm{a}-\mathrm{e}$ mit $\mathrm{NaBH}_{4}$ in EtOH verliefen quantitativ aber mit nur geringer Stereoselekton. Das Isomerenverhaltmis reichte von 59/41 bis 70/30 und wurde liquidchromatographisch aus den Rohproben bestımmt. In allen Fällen bildete $(R, R)-3$ das Hauptdiastereomer Die reinen Stereowsomere wurden nach săulen- bzw. mitteldruckchromatographischer Trennung - in annehmbaren Ausbeuten (siehe Exp -Teil) - erhalten und durch Elementaranalysen, IR, MS und ${ }^{1}$ H-NMR charaktenisiert.

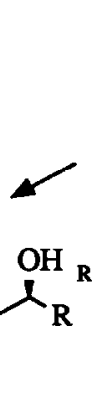

$(R, R)-3$<smiles>[R]C(=O)CC1CCCN1</smiles><smiles>[R]C(C)=O</smiles>

$(R)-1$

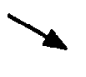<smiles>[R]C(=O)N1CCC[C@H]1C[C@@H]([R])O</smiles>

$(R, S)-4$<smiles>[R]C(=O)CC1CCCN1</smiles><smiles>[R]C([13CH3])=O</smiles>

(S)-2

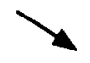

$(S, R)-6$

Tab. 1 Be1 der Reduktıon von $(R)-1 \mathrm{a}-\mathrm{e}$ und $(S)$-2b-d erzielte Diastereoselektıvitäten $(R, R)-3 /(R, S)-4$

$(S, S)-5 /(S, R)-6$

\begin{tabular}{|c|c|c|c|c|c|c|}
\hline $\mathbf{R}$ & $\mathrm{NaBH}_{4}{ }^{\mathrm{a})}$ & $\mathrm{L}_{1}\left(\mathrm{O}^{t} \mathrm{Bu}\right)_{3} \mathrm{AlH}^{\mathrm{b}}$ & $\mathbf{K}\left({ }^{s} \mathrm{Bu}\right)_{3} \mathrm{BH}^{\mathrm{c})}$ & $\mathrm{NaBH}_{4}{ }^{\mathrm{a})}$ & $\mathrm{L}\left(\mathrm{O}^{t} \mathrm{Bu}\right)_{3} \mathrm{AlH}^{\mathrm{b})}$ & $\mathrm{K}\left({ }^{s} \mathrm{Bu}\right)_{3} \mathrm{BH}^{\mathrm{c})}$ \\
\hline a) $-\mathrm{C}\left(\mathrm{CH}_{3}\right)_{3}$ & $70 / 30$ & $93 / 7$ & $\overline{-\ldots}$ & $-\cdots$ & $-\ldots$ & $\overline{---}$ \\
\hline b) $-\mathrm{C}_{6} \mathrm{H}_{5}$ & $59 / 41$ & $85 / 15$ & $3 / 97^{d)}$ & $64 / 36$ & $98 / 2$ & $2 / 5^{d)}$ \\
\hline c) $-\mathrm{C}_{6} \mathrm{H}_{4}-4-\mathrm{Cl}$ & $66 / 34$ & $73 / 27$ & $8 / 92$ & $58 / 42$ & $>95 / 5$ & $2 / 5^{d)}$ \\
\hline d) $-\mathrm{C}_{6} \mathrm{H}_{4}-4-\mathrm{OMe}$ & $61 / 39$ & $78 / 22$ & $2 / 98$ & $65 / 35$ & $95 / 5$ & $1 / 4^{d)}$ \\
\hline e) $-\mathrm{C}_{6} \mathrm{H}_{3}-3-\mathrm{OMe}$ & $63 / 37$ & $86 / 14$ & $4 / 96$ & --- & --- & $\cdots$ \\
\hline
\end{tabular}

a) EtOH, RT

b) THF, $O^{\circ} \mathrm{C}$

c) THF, $-78^{\circ} \mathrm{C}$

d) Durch $400 \mathrm{MHz}{ }^{1} \mathrm{H}-\mathrm{NMR}$ bestummt

An einem ahnlıchen System war beobachtet worden, daß sich die Diastereoselektıvität durch Einsatz von 
$\mathrm{LiAlH}_{4}$ deutlich verbessern läBt ${ }^{15}$. Das traf auch hier zu. Die Reduktion von $(R)$-1d mit $\mathrm{LiAlH}_{4}$ verlief mit einer Diastereoselektıviăt von 73/27 ( $R, R)-3 \mathrm{~d} /(R, S)-4 d$, Tab 2 a). An $(R)-1 d$ haben wir zur Steigerung der Stereoselektivität weitere Reduktionsmittel gerestet. Mit $\mathrm{Li}_{1}\left(\mathrm{O}^{t} \mathrm{Bu}\right)_{3} \mathrm{AlH}$ in THF (bei $\mathrm{O}^{\circ} \mathrm{C}$ ) heB sich eine Diastereoselektıvität von 78/22 (Tab 2 b) erzielen. Beim Austausch von THF durch $\mathrm{Et}_{2} \mathrm{O}$ blieb dieses Verhàltnıs unverändert, wăhrend mit Toluol als Losungsmittel (Tab. 2 e), sowie be1 Vernngerung der Reaktionstemp auf $-40^{\circ}$ (Tab. 2 d) eine genngfügige Verschlechterung eintrat Ein gleichartiger Temperatureffekt war auch bei der Reaktion mit $\mathrm{L}(\mathrm{OMe})_{3} \mathrm{AlH}$ zu verzeichnen, die auch hinsichtlich der Diastereoselektıvität den Umsetzungen mit $\mathrm{Ll}\left(\mathrm{O}^{\prime} \mathrm{Bu}\right)_{3} \mathrm{AlH}$ sehr ăhnlıch war (Tab $2 \mathrm{f}$ und $\mathrm{g}$ )<smiles>[R2]C(C)=O</smiles><smiles>[R]C(=O)N1CCC[C@H]1CC(=O)c1ccc(OC)cc1</smiles>

(R)-1d

Tab. 2 Stereoselektivitaten bei der Umsetzung von $(R)$-1d mit verschiedenen Reduktionsmitteln

$$
\text { Reaktionsbedingungen } \alpha
$$$$
(R, R)-3 \mathrm{~d} /(R, S)-4 \mathrm{~d}^{\beta}
$$

Umsatz

(Red mitrel/Lös mittel/T)

\begin{tabular}{|c|c|c|}
\hline a) $\mathrm{L} 1 \mathrm{AlH}_{4} / \mathrm{THF} /-78^{\circ} \mathrm{C}$ & $73 / 27$ & $95 \%$ \\
\hline b) $\mathrm{L}$ ( $\left(\mathrm{O}^{\mathrm{C}} \mathrm{Bu}\right)_{3} \mathrm{AlH} / \mathrm{THF} / \mathrm{O}^{\circ} \mathrm{C}$ & $78 / 22$ & $98 \%$ \\
\hline c) - " - Ether $/ \mathrm{O}^{\circ} \mathrm{C}$ & $78 / 22$ & $98 \%$ \\
\hline d) - " - THF $/-40^{\circ} \mathrm{C}$ & $60 / 40$ & $80 \%$ \\
\hline e) -" - Toluol $/ 0^{\circ} \mathrm{C}$ & $74 / 26$ & $98 \%$ \\
\hline f) $\mathrm{L} 1(\mathrm{OMe})_{3} \mathrm{AlH} / \mathrm{THF} /-78^{\circ} \mathrm{C}$ & $76 / 24$ & $90 \%$ \\
\hline g) $-" . \quad \quad / \mathrm{THF} /-15^{\circ} \mathrm{C}$ & $79 / 21$ & $90 \%$ \\
\hline h) $\mathrm{LlEt}_{3} \mathrm{BH} / \mathrm{THF} /-78^{\circ} \mathrm{C}$ & $68 / 32$ & $90 \%$ \\
\hline 1) - " - Toluol $/-78^{\circ} \mathrm{C}$ & $76 / 24$ & $95 \%$ \\
\hline J) -"- $15-$ Krone- $5 / \mathrm{THF} /-78^{\circ} \mathrm{C}$ & $60 / 40$ & $98 \%$ \\
\hline k) $\mathrm{D}_{1} \mathrm{BalH} / \mathrm{THF} /-78^{\circ} \mathrm{C}$ & $24 / 76 \gamma$ & $90 \%$ \\
\hline 1) $\mathrm{Ll}\left({ }^{\mathrm{s}} \mathrm{Bu}\right)_{3} \mathrm{BH} / \mathrm{THF} /-78^{\circ} \mathrm{C}$ & $7 / 93$ & $95 \%$ \\
\hline m) - " - Toluol $/-78^{\circ} \mathrm{C}$ & $14 / 86$ & $90 \%$ \\
\hline n) $\mathrm{K}\left({ }^{\mathrm{s}} \mathrm{Bu}\right)_{3} \mathrm{BH} / \mathrm{THF} /-78^{\circ} \mathrm{C}$ & 2/98 & $90 \%$ \\
\hline o) " Toluol/ $-78^{\circ} \mathrm{C}$ & $3 / 97$ & $90 \%$ \\
\hline
\end{tabular}

a) Die Losung des Ketons wurde mit 1 0-1 2 Aquiv Reduktionsmittel versetzt, Endkonzentration ca $0067 \mathrm{~mol} /$, Reaktionsdauer 1-2h. Abweichungen von diesen Bedingungen sind angegeben

B) Durch HPLC bestummt

y) Nebenprodukt mitentstanden

b, c) 32 Aquiv, Reaktionsdauer $16 \mathrm{~h}$

d) 32 Aquiv, Reaktionsdauer $48 \mathrm{~h}$

e) 32 Aqıv, Reduktıonsmittel nıcht gelöst, Reaktıonsdauer 2 d

J) 1 Aquiv Kronenether, inverse Zugabe

Um zu prufen, ob die mit $\mathrm{NaBH}_{4}$ und mit den Alanaten beoabachtete diastereofaciale Selektıvitä́t das Resultat einer Chelat-Kontrolle (s u ) sein könnte, wurde $(R, R)$-1d als nächstes mit DiBalH reduziert In der 
Tat verlief diese Reduktion (DiBalH, THF, $-78^{\circ} \mathrm{C}$ ) zugunsten von $(R, S)-4 \mathrm{~d}((R, R)-3 \mathrm{~d} /(R, S)-4 \mathrm{~d}=24 / 76$, Tab. 2 k) Bei Einsatz von $\mathrm{K}\left({ }^{\circ} \mathrm{Bu}\right)_{3} \mathrm{BH}$ in THF erreichte de Stereoselektion bel gleicher Induktionsnichtung sogar ds $=2 / 98\left(-78^{\circ} \mathrm{C}\right.$, Tab. $\left.2 \mathrm{n}\right)$ an. Daraufhun wurden auch die tibngen Amidoketone mit $\mathrm{Li}\left(\mathrm{O}^{\prime} \mathrm{Bu}\right)_{3} \mathrm{AlH}$ und $\mathrm{K}\left({ }^{\mathrm{B} u}\right)_{3} \mathrm{BH}$ reduziert. Das führte zu vergleıchbaren Resultaten. Mit $\mathrm{L}_{1}\left(\mathrm{O}^{\mathrm{t}} \mathrm{Bu}\right)_{3} \mathrm{AlH}$ entstanden aus $(R)-1$ bevorzugt die $\mathrm{R}, \mathrm{R}$-konfigunerten Produkte $(R, R)-3$ und $\mathrm{mit} \mathrm{K}\left({ }^{5} \mathrm{Bu}\right)_{3} \mathrm{BH}$ hochstereoselektiv die (R,S)-4-Diastereomere (siehe Tab. 1).

Der Reaktıonsverlauf wird im wesentlichen durch das ningständıge Chıralıtätszentrum in einer 1,3-asymmetrischen Induktion bestummt, wie dre Ergebnisse bei der Reduktion von (S)-2 beweisen. Bei ( $S$ )-2 entsteht wie bet $\left.(R)-1 \mathrm{mit} \mathrm{K(}{ }^{{ }^{8} \mathrm{Bu}}\right)_{3} \mathrm{BH}$ bevorzugt das unlike- $((S, R)-6)$ und $\mathrm{mit}^{\mathrm{NaBH}_{4}}$ bzw $\mathrm{Ll}_{1}\left(\mathrm{O}^{\mathrm{t} B u}\right)_{3} \mathrm{AlH}$ das like-konfigurierte Produkt ( $(S, S)-5$, siehe Tab 1)

Außer be1 den $\mathrm{NaBH}_{4}$-Reduktionen (siehe Exp-Teil) wurden die Ausbeuten stellvertretend noch fur zwe1 $\mathrm{K}\left({ }^{\mathrm{s}} \mathrm{Bu}\right)_{3} \mathrm{BH}-$ Reduktionen und zwar von $(R)$-1c und $(R)-1 d$ bestummt Sie lagen bei 665 bzw $63 \%$ fur die durch Kristallisation gewonnenen isomerenreinen Verbindungen $(R, S)-4 \mathrm{c}$ bzw. $(R, S)-4 \mathrm{~d}$.

\section{Stereomodell}

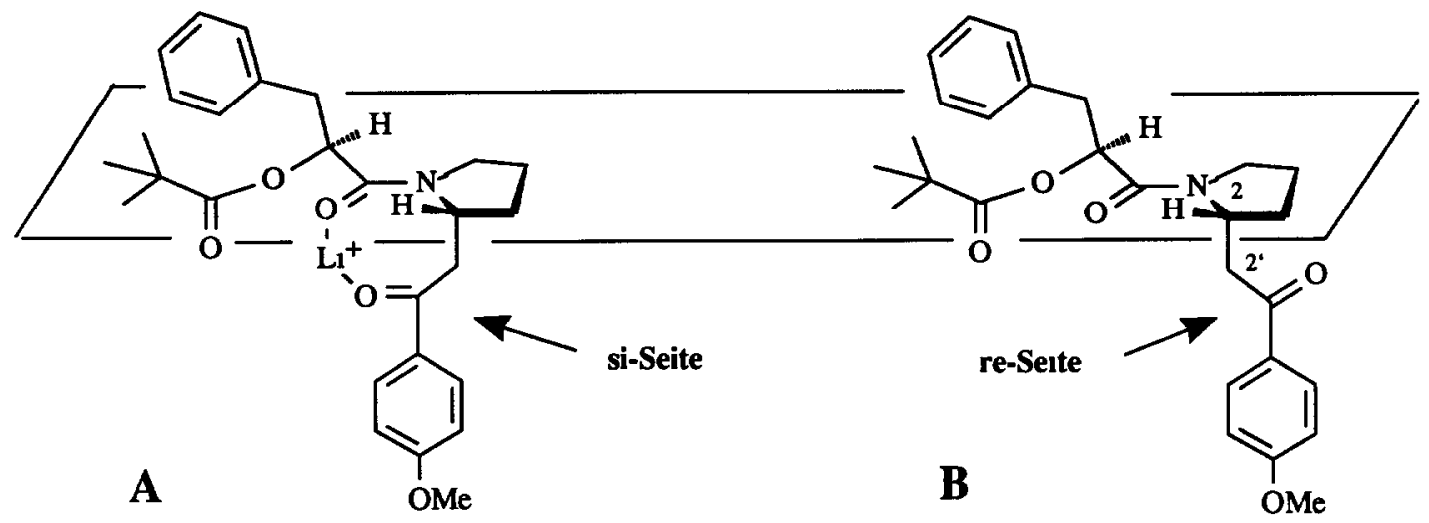

Abb. 1 Chelat- (A) und Nicht-Chelat-Modell (B)

Die Vorzugskonformation von Pyrrolıdınamiden mit einem Substituenten in $\alpha$-Stellung (zum N-Atom) ist so, $\mathrm{da}$ bezuglich der Rotameren um die Amidbindung die Z-Form dominiert ${ }^{16}$ Außerdem durfte der Substituent in $\alpha$-Stellung mehr eıne "axıale" als eıne "äquatoriale" Stellung einnehmen, der sterischen Wechselwirkungen mit dem N-Acylrest wegen Aus den $400 \mathrm{MHz}-{ }^{1} \mathrm{H}-\mathrm{NMR}$-Spektren von $(R)-1$ und $(S)-2$ ist ersichtlich, daß von den Rotameren um die Amıdbindung eines deutlich vorherrscht

Als Erklärung fur den stereochemischen Verlauf der $\mathrm{NaBH}_{4}$ - und $\mathrm{L} 1\left({ }^{\mathrm{B}} \mathrm{BuO}\right)_{3} \mathrm{AlH}-\mathrm{Reduktionen}$ (von $(R)-1$ und analog von (S)-2) bietet sich danach das Chelat-Modell ${ }^{17}$ in Abb 1 A an, in dem die Amido- und Seitenkettencarbonyl-Funktionen das Kation aus dem Reduktionsmittel einschließen ${ }^{18}$ (moghcherweise auch unter Betelligung des Esters) Be1 dem so gebildeten "Achtrnng" ist die konvexe Vorderseite leichter zuganglich Auf sie muß auch der Angnff erfolgen, damit die als Hauptprodukte beobachteten like-konfigunerten Verbindungen $(R, R)-3$ (bzw $(S, S)$-5) gebildet werden 
Mit dem Nicht-Chelat-Modell Abb 1 B kann die Entstehung der unlike-konfigurierten Produkte (R,S)-4 (bzw. $(S, R)-6)$ gedeutet werden Dann ist die re-Seite des Ketons einem Angniff durch das Hydndreagens leichter zugànglich

Fur dieses Modell sprechen die ${ }^{1}$ H-NMR-Spektren der Amidoketone, die einen gewissen RuckschluB auf die Orientuerung der Phenacylseitenkette zulassen Danach ist die Beweglichkeit der Phenacylseitenkette bzgl der C-2-C-2-Achse stark eingeschränkt und Amudstuckstoff und Carbonylkohlenstoff stehen entweder in synclinaler oder antiperiplanarer Beziehung $\left(\mathrm{J}_{2,2},-3 \mathrm{bzw},-12.5 \mathrm{~Hz}\right)$. Die synclinale Anordnung ist wegen der damit verbundenen stenschen Wechselwirkungen mit dem N-Acylrest sehr unwahrscheinlich Die Vorzugskonformation der Amidoketone ( $R$ )-1 durfte folglich in etwa der in Abb. 1 B entsprechen (sinngemáB gilt dies auch fur (S)-2).

Die Ergebnisse unserer Reduktionsversuche stutzen diese Vorstellung, wonach die diastereofaciale Selektıvität eine Funktion von "Chelat-" und "Nicht-Chelat-Kontrolle" darstellt. DaB die Umsetzungen mit Lithiumaluminumhydnd-Reagenzien (Tab. 2 a-g) unter Chelatkontrolle ablaufen, was zu den (R,R)- bzw (S,S)-konfigurierten Produkten fuhrt (siehe Tab 1 und 2 a-g), kann zwanglos mit der Anwesenheit von $\mathrm{Li}^{+}$-Ionen erklărt werden. $\mathrm{Da} \mathrm{DiBalH}$ keine Chelat-Bildung erlaubt, tritt damit die entgegengesetzte Induktionsrichtung auf (Tab. 2 k) Einen Sonderfall bilden die Reduktionen mt den ( $\left.{ }^{8} \mathrm{Bu}\right)_{3} \mathrm{BH}-$ Reagenzien (Tab 2 1-o), die trotz der vorhandenen $\mathrm{Li}^{+}$- bzw $\mathrm{K}^{+}$-Ionen unter unlike-Induktion - derselben wie ber DiBalH - ablaufen Dieses Verhalten - die zur $\mathrm{NaBH}_{4}$-Reduktion entgegengesetzte Stereochemie ist kein Einzelfall ${ }^{19}$ durfte auf das hohe Reduktionspotential der Alkylborhydride zuruckzufuhren sein Da dadurch sicherlıch die Reduktion auch von solchen Carbonylgruppen, die nicht durch Anlagerung eines Kations aktıviert ${ }^{20}$ sind, erleichtert wird, durfte das Reduktionsmittel von der sterisch gehinderten Chelatform auf die Nicht-Chelat-Form ausweichen

$\mathrm{Daß}$ sterısche Hinderung eine Rolle spielt, wird durch die Umsetzung mit dem räumlich weniger anspruchsvollen $\mathrm{LiEt}_{3} \mathrm{BH}$ bestangt, bet der weder das Produkt der Chelat-Kontrolle dominert (Tab $2 \mathrm{~h}$ und 1)

Die Abhangigkeit der Diastereoselektuvitat vom Losungsmittel und Gegenion 1st ebenfalls sehr aufschlußreich Wird THF durch Toluol ersetzt, erhöht sich sowohl bei der Reduktion mit $\mathrm{LiEt}_{3} \mathrm{BH}$, als auch mit L1- wie K( $\left.{ }^{(} \mathrm{Bu}\right)_{3} \mathrm{BH}$ der Anteil des chelat-kontrollierten Produkts $(R, R)-3 \mathrm{~d}$ (vgl Tab $2 \mathrm{~h}$ mit 1, 1 mit m und $\mathrm{n}$ mit o) Das genaue Gegenteil tritt beim Austausch von $\mathrm{Li}^{+}$durch $\mathrm{K}^{+}$auf (vgl Tab 21 mit $\mathrm{n}$ und $\mathrm{m}$ mit $\mathrm{o}$ ) $\mathrm{Da}$ von diesen Maßnahmen die einen die Chelatbildungstendenz erhohen (die geringere Solvenspolantat), die anderen verningern (Folge der genngeren Lewisaciditat von $\mathrm{K}^{+}$), entsprechen die beobachteten Effekte genau dem Modell

Weshalb die Reduktion von $(R)$-1d mit $\mathrm{LiEt}_{3} \mathrm{BH}$, dem zur Komplexierung des $\mathrm{Li}^{+}$-Ions ein Kronenether zugesetzt war ${ }^{21}$, uberwiegend das chelatkontrollierte Produkt $(R, R)$-3d ergab (Tab $2 \mathrm{~J}$ ), ist unklar

\section{Reduktıon eines Amidoketons mit achiralem N-Acylrest}

Um etwas uber den Gultngkeıtsbereich unseres Modells zu erfahren, wurde (rac)-9, das einen achiralen N-Acylrest besitzt, in diese Untersuchungen miteinbezogen Zur Darstellung von (rac)-9 wurde wie im Formelschema angegeben vorgegangen 
Die Reduktion von ( $r a c$ )-9 mit $\mathrm{NaBH}_{4}$ und mit LiEt 3 BH ergab nur eine gennge Stereoselektion mit (rac)-11 als Hauptprodukt (Tab 3 a und c) Mit K( $\left.{ }^{(B)}\right)_{3}$ BH lag die Stereoselektion deutlich höher, mit 19.81 (Tab 3 d) und (rac)-11 war - wie fur Nicht-Chelat-Kontrolle zu erwarten - das dominierende Stereo1somer Das entspncht etwa dem Mittelwert aus den bel $(S)-2$ und $(R)-1$ bei den $\mathrm{K}\left({ }^{3} \mathrm{Bu}\right)_{3} \mathrm{BH}-\mathrm{Reduktionen}$ gefundenen Stereoselektuvitäten<smiles>CC(C)(C)C(=O)N1CCCC1CC(=O)c1ccccc1</smiles>

(rac)-9

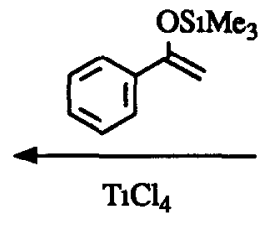

$\sim_{1}$<smiles>[2H][C@H](C[C@@H]1CCCN1C(=O)C(C)(C)C)c1ccccc1</smiles>

(rac)-10<smiles>[2H][C@H](C[C@@H](O)c1ccccc1)C1CCCN1C(=O)C(C)(C)C</smiles>

(rac)-11<smiles>COC1CCCN1C(=O)C(C)(C)C</smiles>

(rac)-8<smiles>COC(=O)OC1CCCN1C(=O)C(C)(C)C</smiles>

$(S)-7$

Tab. 3 Stereoselektıvităten bei der Reduktion von (rac)-9

Reaktionsbedingungen ( $\mathrm{rac})-10 /(\mathrm{rac})-11$
a) $\mathrm{NaBH}_{4} / \mathrm{ETOH} / \mathrm{RT}$
$46 / 54$
b) $\mathrm{Ll}\left(\mathrm{O}^{\mathrm{t}} \mathrm{Bu}\right)_{3} \mathrm{AlH} / \mathrm{THF} / \mathrm{O}^{\circ} \mathrm{C} \quad 60 / 40$
c) $\mathrm{LIEt}_{3} \mathrm{BH} / \mathrm{THF} /-78^{\circ} \mathrm{C}$
d) $\mathrm{K}\left({ }^{\mathrm{s}} \mathrm{Bu}\right)_{3} \mathrm{BH} / \mathrm{THF} /-78^{\circ} \mathrm{C}$

Die be1 (R)-1 und (S)-2 unter Nicht-Chelat-Kontrolle beobachtete hohe Stereodifferenzierung ist folglich nucht eine Besonderheit dieses einen N-Acylrestes, wenngleıch er durch seine Chiralitát das Induktionsvermögen des nungständıgen Chıralıtätszentrums be1 $(R)-1$ verstärkt und be1 $(S)$-2 abschwácht Dieser Effekt von "matched" und "mismatched"22. par ze1gt sich auch be1 den chelat-kontrollierten Reduktionen (mit $\mathrm{Li}\left(\mathrm{O}^{\prime} \mathrm{Bu}\right)_{3} \mathrm{AlH}$, vgl Tab 2), jedoch mit entgegengesetztem Vorzeichen, denn in diesem Fall liefert ( $S$ )-2 die höhere Selektıvitàt.

Das Ergebnis bei der Reduktion von (rac)-9 mit $\mathrm{Ll}\left(\mathrm{O}^{t} \mathrm{Bu}\right)_{3} \mathrm{AlH}$ weicht davon nun mit 60/40 deutlich nach unten ab Fur die chelat-kontrollierte Induktion 1st demnach das churale Auxiliar von entscheidender Bedeutung, womoglıch wegen der Esterfunktion, indem diese zur Festıgung des Chelats beitragt

\section{Aminoalkohole}

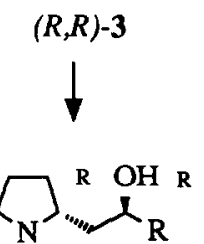

$\mathrm{H}$

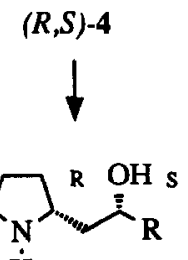

$\dot{\mathbf{H}}$

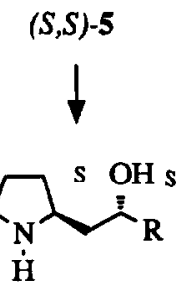

$(S, S)-12$
$(S, R)-6$

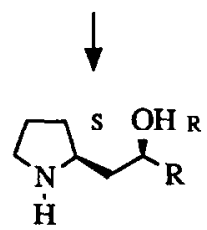

$(S, R)-13$ 
Tab. 4 Ausbeuten und $[\alpha]_{578}$-Werte der Aminoalkohole $(R, R)-12 \mathrm{a}-\mathrm{d},(R, S)-13 \mathrm{a}-\mathrm{d},(S, S)-12 \mathrm{~b}-\mathrm{d}$ und $(S, R)-13 \mathbf{b}-\mathbf{d}$

$(R, R)-12 \mathrm{a}-\mathrm{d}$

$\mathbf{R}$

Ausb $[\%] \quad[\alpha]_{578}$

$(R, S)-13 a-d$

Ausb.[\%] $[\alpha]_{578}$
$(S, S)-12 b-d$

Ausb $[\%] \quad[\alpha]_{578}$
$(S, R)-13 b-\mathrm{d}$

Ausb [\%] $[\alpha]_{578}$

\begin{tabular}{lllllllll}
\hline a) $-\mathrm{C}\left(\mathrm{CH}_{3}\right)_{3}$ & 71 & $+24.7^{\circ}$ & 51 & $+8^{\circ}$ & -- & -- & -- & - \\
b) $-\mathrm{C}_{6} \mathrm{H}_{5}$ & 69 & $+36.8^{\circ}$ & 51 & $-12^{\circ}$ & 55 & $-36.1^{\circ}$ & 54 & $+118^{\circ}$ \\
c) $-\mathrm{C}_{6} \mathrm{H}_{4}-4-\mathrm{Cl}$ & 77 & $+315^{\circ}$ & 76 & $-102^{\circ}$ & 61 & $-343^{\circ}$ & 77 & $+93^{\circ}$ \\
d) $-\mathrm{C}_{6} \mathrm{H}_{4}-4-\mathrm{OMe}$ & 74 & $+332^{\circ}$ & 72 & $-133^{\circ}$ & 63 & $-312^{\circ}$ & 65 & $+107^{\circ}$ \\
\hline
\end{tabular}

Die Aminoalkohole 12 bzw 13 heßen sich in Ausbeuten von 51 bis 77\% durch mehrstundiges Erhitzen der entsprechenden Amıdoalkohole mit Tetrabutylammoniumhydroxid (TBAH) in THF erhalten

Die Hydrolyse von ( $\mathrm{rac}$ )-11 mit TBAH zum Aminoalkohol diente der Konfiguratoonszuordnung, das Produkt wurde als de Racematform von $(R, S)-13 /(S, R)-13$ identufiziert

$\gamma$-Amınoalkohole neigen dazu, intramolekulare Wasserstoffbruckenbındungen auszubilden mit der Folge, daß 1,3-oxazınartıge Strukturen mit sesselähnlıcher Konformatıon entstehen ${ }^{\text {tb }}$

Nach IR-Untersuchungen an $(R, R)-12$, bei denen sich die Lage der $\mathrm{OH}-\mathrm{Bande}\left(3200 \mathrm{~cm}^{-1}\right.$ in $\left.\mathrm{CCl}_{4}\right)$ als konzentrationsunabhängıg erwies, 1st dies auch hier der Fall

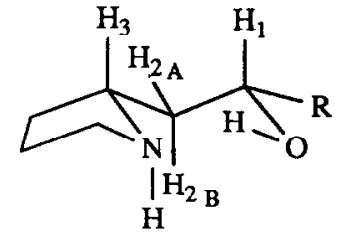

$(R, R)-12$

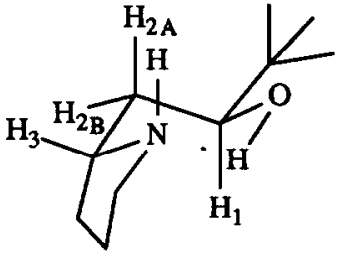

$(R, S)-13 \mathbf{a}$

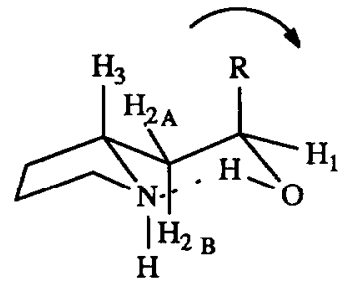

$(R, S)-13 \mathrm{~b}-\mathrm{d}$
Tab. 5 Ausgewahlte Kopplungskonstanten von $(R, R)-12 \mathrm{a}-\mathrm{d} \quad(1 \mathrm{n} \mathrm{Hz})$

\begin{tabular}{lllll}
$(R, R)-12$ & $\mathrm{~J}_{1,2 \mathrm{~B}}$ & $\mathrm{~J}_{1,2 \mathrm{~A}}$ & $\mathrm{~J}_{2 \mathrm{~B}, 3}$ & $\mathrm{~J}_{2 \mathrm{~A}, 3}$ \\
\hline a & 106 & 15 & 116 & 31 \\
b & 106 & 22 & 114 & 34 \\
c & 106 & 18 & 114 & 31 \\
d & 103 & 22 & 112 & 33 \\
\hline
\end{tabular}

Tab. 6 Ausgewahlte Kopplungskonstanten von $(R, S)-13 \mathrm{a}-\mathrm{d}$ (in $\mathrm{Hz}$ )

\begin{tabular}{lcccc}
$(R, S)-13$ & $\mathrm{~J}_{1,2 \mathrm{~B}}$ & $\mathrm{~J}_{1,2 \mathrm{~A}}$ & $\mathrm{~J}_{2 \mathrm{~A}, 3}$ & $\mathrm{~J}_{2 \mathrm{~B}, 3}$ \\
\hline a & 20 & 107 & 42 & 41 \\
b & $62^{\mathrm{b}}$ & $42^{\mathrm{b}}$ & $--^{\mathrm{a}}$ & $--^{\mathrm{a}}$ \\
c & $78^{\mathrm{b}}$ & $29^{\mathrm{b}}$ & $--^{\mathrm{a}}$ & $--^{\mathrm{a}}$ \\
d & $51^{\mathrm{c}}$ & $51^{\mathrm{c}}$ & $-^{\mathrm{a}}$ & $--^{\mathrm{a}}$ \\
\hline
\end{tabular}

a) Kopplungskonstanten $<7 \mathrm{~Hz}$

b) Zuordnung kann vertauscht sein

c) In $D_{6}$-Benzol 44 bzw $59 \mathrm{~Hz}$ 
Durch die $400 \mathrm{MHz}-{ }^{1} \mathrm{H}-\mathrm{NMR}-\mathrm{Spektren}$ von $(R, R)$-12a-d wird dies bestätigt. Die vicinalen Kopplungskonstanten zwischen 2- $\mathrm{H}$ und 1-H bzw 3-H weisen die fur eine Sesselanordnung typischen Werte auf Be1 einem der beiden 2-H-Protonen (2B-H) treten Kopplungskonstanten von $-105 \mathrm{bzw}-115 \mathrm{~Hz}\left(\mathrm{~J}_{1,2 \mathrm{~B}}\right.$ und $\left.\mathrm{J}_{2 \mathrm{~B}, 3}\right)$ und beim anderen $(2 \mathrm{~A}-\mathrm{H})$ von $-2 \mathrm{bzw}-3 \mathrm{~Hz}\left(\mathrm{~J}_{1,2 \mathrm{~A}}\right.$ und $\left.\mathrm{J}_{2 \mathrm{~A}, 3}\right)$ zu den Nachbarprotonen 1-H bzw 3-H auf Fur die Protonen 1-H, 3-H und 2B-H ergibt sich daraus eine axiale, fur 2A-H eine äquatoriale Stellung Zur Sicherung wurden an $(R, R)-12 \mathrm{c}$ noch NOE-Messungen vorgenommen. Sättgung des 1 -H-Signals hatte eine Intensıtätssteıgerung beı 3-H zur Folge Somit stehen sıch diese Protonen räumlıch nahe, wie es bei einer Sesselgeometrie sein muß

Hieraus folgt, daß die Amnoalkohole $(R, R)-12$ und die dazu enantomeren Verbindungen ( $S, S)-12$ like konfigunert sind ${ }^{23}$ Die angegebene absolute Konfiguration folgt aus derjenigen der Ausgangsprodukte Die dazu diastereomeren Verbindungen sind zwangslaufig $(R, S)$-13 bzw $(S, R)$-13

$\mathrm{Da}$ diese Verbindungen unlike-konfigunert sind, kommt auch in den ${ }^{1} \mathrm{H}-\mathrm{NMR}$-Spektren zum Ausdruck Die 2-H-Protonen von $(R, S)$-13a koppeln - anders als bei $(R, R)-12$ - beide (2A-H und 2B-H) mit einer Konstanten von nur $-4 \mathrm{~Hz}$ mit 3-H Die Kopplungen zu 1-H, $\mathrm{J}_{2 \mathrm{~A}, 1}$ und $\mathrm{J}_{2 \mathrm{~B}, 1}$, betragen dagegen $107 \mathrm{bzw} 20 \mathrm{~Hz}$ Demnach $\mathrm{muB}(R, S)$-13a eıne der Formel $(R, S)$-13a entsprechende Konformation mit cis-verknupften Ringen einnehmen

Die phenyl-substituierten Amınoalkohole $(R, S)$-13b-d zeigen ein davon abweichendes Verhalten. Aus den Kopplungskonstanten zwischen den 2-H- und 1-H-Protonen 1st zu schließen (siehe Tab 6), daß die Phenylgruppe micht rein axial, oder rein equatorial steht Sterische Wechselwirkungen des Phenylringes mit 3-H haben vermutlich zu einer Verzerrung des Sessels gefuhrt ${ }^{24}$ (siehe Pfell in der Formel), oder eine ringoffene Form hat an Bedeutung gewonnen Darauf weist zumindest die bel $(R, S)$-13d beobachtete Losungsmıttelabhängıgkett der Kopplungskonstanten hın (Tab 6)<smiles>O=C(O)[C@H](O)Cc1ccccc1</smiles>

Tab. 7 Enantiomerenreinhelt von (S)-14

\begin{tabular}{cc}
$\begin{array}{c}(S)-14 \\
\text { Probenherkunft }\end{array}$ & ee $(\%)$ \\
\hline$(R, R)-3 \mathrm{~b}$ & 988 \\
$(R, S)-4 \mathrm{~d}$ & 982 \\
$(S, R)-6 \mathrm{c}$ & 978
\end{tabular}

Beı der Hydrolyse der Amıdoalkohole wird Phenylmilchsaure (S)-14 freı Wur haben sie in einigen Fällen zuruckgewonnen und nach einem bekannten $\operatorname{Verfahren}^{25}$ (mit (S)-1-Naphthylethylamin als chiralem Derivatısierungsreagens) ihre Enantiomerenreinheit bestımmt Die Ergebnisse sind in Tab 7 zusammengestellt. Der medngste Wert lag bei $978 \%$ ee Zieht man ihn und die Tatsache in Betracht, daß zur Aminoalkohol-Darstellung nur Amidoketone mit de $\geq 98 \%$ verwendet wurden, mussen die Aminoalkohole einen ee-Wert $>95 \%$ aufweisen

Zur Zeıt untersuchen wir die pharmakologischen Eigenschaften dieser Verbindungen und thre Abhangigkeit von der Stereochemie 


\title{
EXPERIMENTELLER TEIL
}

Schmp (nicht korr) Apparatur nach Dr Tottol. - ${ }^{1}$ H-NMR-Spektren T-60 und A-60 (Vanan), AM 360 (Bruker), JNM-GX 400 (Jeol), 8-Skala (ppm), TMS int. Stand; Auswertung der 'H-NMR-Spektren nach Năherung 1 Ordnung; Rotationsisomere sind entsprechend der beobachteten Verteilung angegeben, sofern sie als solche eindeutig zu erkennen waren, ansonsten wurde das Signal des Hauptrotamers $=1 \mathrm{H}$ gesetzt - MS CH 7 (Varıan) - IR-Spektren Acculab 6 (Beckman), 710 B und 881 Perkn-Elmer, Flüssigketten und Öle als Film, Feststoffe als KBr-PreBlunge - Optische Drehungen: Lichtelektnsches Polarimeter Ze1ss, $0.5 \mathrm{dm}$ Rohr - CHN-Analysen' CHN-Rapid (Heraeus) - SC. Flash-Chromatographie, Kueselgel 60 (0.040-0 063 mm) Zentrifugalchromatographie: Chromatotron (Hamson Research), Kieselgel 60 PF 254, gipshaltg

Alle Schutzgas erfordernden Arbeiten wurden unter Stackstoff durchgefihrt - MPLC. HPLC Pumpe 64 (Knauer, mit präp. Pumpenknopf), Mitteldrucksäule $460 \times 26 \mathrm{~mm}$ und $460 \times 36 \mathrm{~mm}$ mit Vorsäule (Buch1), Kieselgel S1 $60(0.015-0.040 \mathrm{~mm})$, UV-Detektor $254 \mathrm{~nm}$ Modell 8201 (Bischoff), Integrator Datamodul (Waters) - HPLC Chromatographiepumpe L6000 (Merck-Hitach1) Brechungsindexdetektor R 401, UV-Detektor 440, $254 \mathrm{~nm}$ (Waters), Integrator D-2000 (Merck-Hitachi), Süulenheizung Knauer High Temperature Oven No. 8900 Series 799; LiChroCart ${ }^{R}$, LiChrosorb ${ }^{R}$ Si60 HPLC-Kartusche (250x4 mm 1 D.) und LiChroCart ${ }^{R}$, LiChrosorb ${ }^{R} S_{1} 60$ Kartuschenvorsăule (25x4 mm 1.D.,Merck); chirale Săule Pirkle-Säule ((R)-N-3,5-Dinitrobenzoylphenylglycin kovalent gebunden, Baker) -Präp.-HPLC. Pumpe, Detektor und Integrator wie bel MPLC, HPLC-Săule Bischoff, LiChrosorb S1 $607 \mu \mathrm{m}$ (460x26 mm 1.D Bischoff), Saulenhe1zung (wie oben) - Elektrolyseapparatur Leistungsgleichnchter $120 \mathrm{~V}, 4-2 \mathrm{~A}, 200 \mathrm{~W}$, ungeteilte Elektrolysezelle $250 \mathrm{ml}$ mit Kuhlmantel, Pt-Netzelektroden zylinderförmig angeordnet, $\varnothing 22$ und $35 \mathrm{~mm}, \mathrm{~h}=$ $50 \mathrm{~mm}$.

\begin{abstract}
Allgemeine Arbeitsvorschriften
AAV 1 - $\mathrm{NaBH}_{4}$-Reduktion

Die zu reduzierende Verbindung ( $1 \mathrm{mmol}$, de> 98\%) wird in EtOH $(18 \mathrm{ml})$ gelöst und sodann unter Eiskuhlung mit einer $\mathrm{NaBH}_{4}$-Suspension $(2,8 \mathrm{mmol}$ in $2 \mathrm{ml} \mathrm{EtOH})$ versetzt Nach 15 min wird auf Raumtemp. erwärmt und noch $16 \mathrm{~h}$ bei dieser Temp geruhrt. Uberschüssiges Reduktionsmittel wird durch tropfenweise Zugabe gesättıgter $\mathrm{NH}_{4} \mathrm{Cl}$-Lösung zerstört (Gasentwicklung') Anschließend wird mit $\mathrm{H}_{2} \mathrm{O}$ verdunnt und das Gemsich bei einer Badtemperatur von $-35^{\circ} \mathrm{C}$ i Vak weitgehendst von EtOH befreit Die verbleibende waßnge Phase wird mit $\mathrm{Et}_{2} \mathrm{O}(4 \mathrm{x})$ extrahiert Die organischen Extrakte werden vereinigt, getrocknet $\left(\mathrm{MgSO}_{4}\right)$ und 1 Vak eingeengt Der Ruckstand wird sc getrennt.
\end{abstract}

AAV 2 - Li(OtBu $)_{3}$ AlH-Reduktion

Die zu reduzierende Verbindung ( $1 \mathrm{mmol}$, de $>98 \%$ ) wrd in absol. THF (15 ml) gelost und unter Eiskuhlung mit $\mathrm{Ll}\left(\mathrm{O}^{\top} \mathrm{Bu}\right)_{3} \mathrm{AlH}\left(32 \mathrm{mmol}\right.$ ) versetzt $\mathrm{Nach}$ einer Reaktionsdauer von $16 \mathrm{~h}$ (bei $\mathrm{O}^{\circ} \mathrm{C}$ ) wird wie unter AAV 1 angegeben bis zur SC-Trennung aufgearbettet

\section{AAV 3 - K('Bu $)_{3}$-BH-Reduktion}

Die zu reduzierende Verbındung ( $1 \mathrm{mmol}$, de $>98 \%)$ wird in absol THF (15 ml) gelöst, dann wird auf $-78^{\circ} \mathrm{C}$ abgekuhlt und mit $\mathrm{K}\left({ }^{\mathrm{B}} \mathrm{Bu}\right)_{3} \mathrm{BH}$ (12 mmol als $1 \mathrm{M}$-Lósung in THF) versetzt. Die Reaktion wird nach $2 \mathrm{~h}$ abgebrochen Dazu wird bei $-78^{\circ} \mathrm{C}$ zunächst tropfenweise $\mathrm{MeOH}$ dann $\mathrm{H}_{2} \mathrm{O}$ zugegeben. Anschließend wird das Gemisch 1 Vak bei einer Badtemp von $-35^{\circ} \mathrm{C}$ weitgehendst von org. Lösungsmitteln befreit Dann wird mit $\mathrm{Et}_{2} \mathrm{O}(4 \mathrm{x})$ extrahiert und nach Trocknen $\left(\mathrm{MgSO}_{4}\right)$ der Extrakte i Vak eingeengt

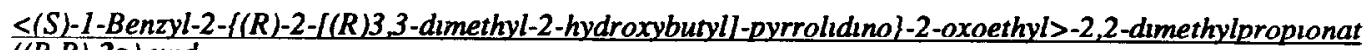
$((R, R)-3 \mathrm{a})$ und

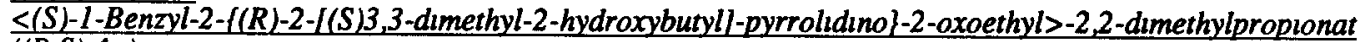
$((R, S)-4 \mathrm{a})$

a) Nach AAV 1 aus $600 \mathrm{mg}(15 \mathrm{mmol})(R)-1 \mathrm{a}^{25} \mathrm{SC}$ n-Hexan/ErOAc $=4 / 6$

$(R, R)$-3a Farblose Kristalle, Schmp $90^{\circ} \mathrm{C},[\alpha]_{546}=+894^{\circ},[\alpha]_{578}=+801^{\circ}\left(\mathrm{c}=151, \mathrm{CHCl}_{3}\right)$, Ausb $298 \mathrm{mg}$ (49 3\%) $-\mathrm{C}_{24} \mathrm{H}_{37} \mathrm{NO}_{4}(4036)$ Ber C $714 \mathrm{H} 924 \mathrm{~N} 35 \mathrm{Gef} \mathrm{C} 716 \mathrm{H} 932 \mathrm{~N} 3.3 \mathrm{Mol}$.-Masse 403 (ms) -IR: $3420,3370,1730,1640,1625 \mathrm{~cm}^{-1}-360 \mathrm{MHz}^{-1} \mathrm{H}-\mathrm{NMR}\left(\mathrm{CDCl}_{3}\right) \cdot 076\left(\mathrm{~s}, 087 \mathrm{x} 9 \mathrm{H},{ }^{\mathrm{t}} \mathrm{Bu}\right), 084(\mathrm{~s}, 013 \times 9 \mathrm{H}$, $\left.{ }^{\mathrm{t}} \mathrm{Bu}\right), 108\left(\mathrm{~s}, 013 \times 9 \mathrm{H}, \mathrm{CO}^{\mathrm{t}} \mathrm{Bu}\right), 121\left(\mathrm{~s}, 0.87 \times 9 \mathrm{H}, \mathrm{CO} \mathrm{CO}^{\mathrm{B} u}\right), 14-175\left(\mathrm{~m}, 6 \mathrm{H}, \mathrm{NCH}_{2} \mathrm{CH}_{2} \mathrm{CH}_{2}, \mathrm{CH}_{2} \mathrm{COH}\right), 262$ (dt, J=7/9Hz, $\left.087 \times 1 \mathrm{H}, \mathrm{NCH}_{2}\right), 302-31\left(\mathrm{~m}, 2 \mathrm{H}, \mathrm{CH}_{2} \mathrm{Ph}\right), 308$ (dd, $\left.\mathrm{J}=3 / 10 \mathrm{~Hz}, 1 \mathrm{H}, \mathrm{CHOH}\right), 3.33-3.40$ (m, $\left.013 \times 1 \mathrm{H}, \mathrm{NCH}_{2}\right), 344-352\left(\mathrm{~m}, 013 \times 1 \mathrm{H}, \mathrm{NCH}_{2}\right), 358(\mathrm{dt}, \mathrm{J}=27 / 9 \mathrm{~Hz}, 087 \times 1 \mathrm{H}, \overline{\mathrm{NCH}}), 403-41(\mathrm{~m}$, $087 \times 1 \mathrm{H}, \mathrm{NCH}), 448-455(\mathrm{~m}, 0.13 \times 1 \mathrm{H}, \mathrm{NCH}), 498(\mathrm{t}, \mathrm{J}=7.5 \mathrm{~Hz}, 087 \times 1 \mathrm{H}, \mathrm{OCHCO}), 538$ (dd, J=5/9Hz, $013 \times 1 \mathrm{H}, \mathrm{OCHCO}), 72-74(\mathrm{~m}, 5 \mathrm{H}, \mathrm{Ph}), \mathrm{OH}$ nicht lokalisiert Rotamerenverhältnis ca. $7 / 1$

$(R, S)-4 \mathrm{a}$ Farblose Kristalle, Schmp $81^{\circ} \mathrm{C},[\alpha]_{546}=+36.1^{\circ},[\alpha]_{578}=+302^{\circ}\left(\mathrm{c}=105, \mathrm{CHCl}_{3}\right)$, Ausb $168 \mathrm{mg}$ (278\%) - $\mathrm{C}_{24} \mathrm{H}_{37} \mathrm{NO}_{4}$ (4036) Ber C $714 \mathrm{H} 924 \mathrm{~N} 35$ Gef C $717 \mathrm{H} 91 \mathrm{~N} 34$ Mol.-Masse 403 (ms) -IR $3370,1735,1640 \mathrm{~cm}^{-1}-360 \mathrm{MHz}^{-1} \mathrm{H}-\mathrm{NMR}\left(\mathrm{CDCl}_{3}\right) 0.85\left(\mathrm{~s}, 9 \mathrm{H},{ }^{\mathrm{t}} \mathrm{Bu}\right), 12\left(\mathrm{~s}, 9 \mathrm{H}, \mathrm{CO}^{\mathrm{B}} \mathrm{Bu}\right), 122-175(\mathrm{~m}$, $5 \mathrm{H}, \mathrm{NCH}_{2} \mathrm{CH}_{2} \underline{\mathrm{CH}}_{2}, \mathrm{CH}_{2} \mathrm{COH}$ ), $182-197\left(\mathrm{~m}, 1 \mathrm{H}, \mathrm{NCH}_{2} \underline{\mathrm{CH}}_{2} \mathrm{CH}_{2}\right.$ ), 2.78 (dt, J=8 /9 5Hz, 1H, NCH ), 297 
(ddd, J=2 4/4 3/10Hz, 1H, CHOH), 316 (d, J=7.6Hz, 2H, CH $\mathrm{Ph}$ ), 3.57 (dt, J=2/9.5Hz, 1H, NCH ), $440-4.48(\mathrm{~m}, 1 \mathrm{H}, \mathrm{NCH}), 4.89$ (d, J=4.3Hz, $1 \mathrm{H}, \mathrm{OH}), 5.09(\mathrm{t}, \mathrm{J}=7.6 \mathrm{~Hz}, 1 \mathrm{H}, \mathrm{OCHCO}), 7.24-733(\mathrm{~m}, 5 \mathrm{H}, \mathrm{Ph})$ HPLC-Analyse (n-Hexan/EtOAC=3/1, 2ml/min); $(R, S)-4 \mathrm{a}(7.7 \mathrm{~min}) /(R, R)-3 \mathrm{a}(15.3 \mathrm{~min}): 30 / 70$. b) Nach AAV 2 aus $40 \mathrm{mg}(0.1 \mathrm{mmol})(R)-1 \mathrm{a}$. Nach $360 \mathrm{MHz}-{ }^{1} \mathrm{H}-\mathrm{NMR}$ : $(R, R)-3 \mathrm{a} /(R, S)-4 \mathrm{a}-93 / 7$.

 $((R, R)-3 \mathbf{b})$ und

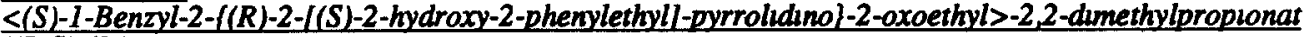 $((R, S)-4 b)$}

a) Nach AAV 1 aus $842 \mathrm{mg}(2 \mathrm{mmol})(R)-1 b^{14}$. SC: $n-H e x a n / E t O A c=6 / 4$

$(R, R)-3 b$ Farblose Knstalle, Schmp. $132^{\circ} \mathrm{C},[\alpha]_{546}=+163.3^{\circ},[\alpha]_{578^{\prime}}=+1411^{\circ}$ (c=1 935, $\mathrm{CHCl}_{3}$ ),Ausb. 381 $\mathrm{mg}(45.0 \%)-\mathrm{C}_{26} \mathrm{H}_{33} \mathrm{NO}_{4}$ (423 6) Bcr. C 73.7 H 7.85 N 3.3 Gef. C $73.6 \mathrm{H} 7.74 \mathrm{~N} 3.4$ Mol.-Masse 423 (ms).-IR 3400, 1720, $1630 \mathrm{~cm}^{-1} .-400 \mathrm{MHz}^{-1} \mathrm{H}-\mathrm{NMR}\left(\mathrm{CDCl}_{3}\right): 1.14\left(\mathrm{~s}, 01 \times 9 \mathrm{H},{ }^{\mathrm{B}} \mathrm{Bu}\right), 121$ (s, 0.9x9H, $\left.1 \mathrm{Bu}\right)$, 1 6-1.9 (m, 4H, $\mathrm{NCH}_{2} \mathrm{CH}_{2} \mathrm{CH}_{2}$ ), 1.72 (ddd, J=2.6/6 2/14.7Hz, $1 \mathrm{H}, \mathrm{CH}_{2} \mathrm{COH}$ ), 1.99 (ddd, $\mathrm{J}=3.8 / 11 / 147 \mathrm{~Hz}$, $\left.1 \mathrm{H}, \mathrm{CH}_{2} \mathrm{COH}\right), 2.72\left(\mathrm{dt}, \mathrm{J}=70 / 95 \mathrm{~Hz}, 0.9 \times 1 \mathrm{H}, \mathrm{NCH}_{2}\right), 3.08-3.2\left(\mathrm{~m}, 2 \mathrm{H}, \mathrm{CH}_{2} \mathrm{Ph}\right), 3.35-3.42(\mathrm{~m}, 0.1 \times 1 \mathrm{H}$, $\mathrm{NCH}_{2}, 354-3.6\left(\mathrm{~m}, 0.1 \times 1 \mathrm{H}, \mathrm{NCH}_{2}\right), 3.63\left(\mathrm{dt}, \mathrm{J}=2.919 .5 \mathrm{~Hz}, 0.9 \times 1 \mathrm{H}, \mathrm{NCH}_{2}\right), 422-4.28(\mathrm{~m}, 0.9 \times 1 \mathrm{H}, \mathrm{NCH})$, $465-47$ (m, 0.1x1H, CHOH), 472 (dd, J=2.6/11Hz, 0.9x1H, CHOH), $472-4.78$ (m, 0.1x1H, NCH), 510 (t, $\mathrm{J}=75 \mathrm{~Hz}, 0.9 \times 1 \mathrm{H}, \mathrm{OCHCO}$ ), 5.42 (dd, $\mathrm{J}=5 / 9 \mathrm{~Hz}, 01 \times 1 \mathrm{H}, \mathrm{OCHCO}), 719-738(\mathrm{~m}, 10 \mathrm{H}, \mathrm{Ph}), \mathrm{OH}$ nicht lokalisiert. Rotamerenverhältnis: ca $9 / 1$

$(R, S)-4 b$ : Farblose Kristalle, Schmp $109-111^{\circ} \mathrm{C},[\alpha]_{546}=+889^{\circ},[\alpha]_{578}=+75.6^{\circ}\left(\mathrm{c}=1.935, \mathrm{CHCl}_{3}\right)$, Ausb. 270 $\mathrm{mg}(319 \%)-\mathrm{C}_{26} \mathrm{H}_{33} \mathrm{NO}_{4}(4236) \mathrm{Ber} \mathrm{C} 737 \mathrm{H} 7.85 \mathrm{~N} 3.3 \mathrm{Gef} \mathrm{C} 738 \mathrm{H} 769 \mathrm{~N} 34 \mathrm{Mol}$-Masse 423 (ms) -IR $3350,1720,1640 \mathrm{~cm}^{-1}-400 \mathrm{MHz}^{-} \mathrm{H}-\mathrm{NMR}\left(\mathrm{CDCl}_{3}\right) 1.22(\mathrm{~s}, 9 \mathrm{H}, \mathrm{Bu}), 1.49-155(\mathrm{~m}, 1 \mathrm{H}$, $\mathrm{NCH}_{2} \mathrm{CH}_{2} \mathrm{CH}_{2}$ ), $161-175\left(\mathrm{~m}, 4 \mathrm{H}, \mathrm{CH}_{2} \mathrm{COH}, \mathrm{NCH}_{2} \mathrm{CH}_{2} \mathrm{CH}_{2}\right.$ ), 1.8-1 95 (m, 1H, NCH $\mathrm{CH}_{2} \mathrm{CH}_{2}$ ), 278 (dt, $\mathrm{J}=73 / 97 \mathrm{~Hz}, 1 \mathrm{H}, \mathrm{NCH}_{2}$ ), $3.15\left(\mathrm{~d}, \mathrm{~J}=7 \mathrm{7Hz}, 2 \mathrm{H}, \mathrm{CH}_{2} \mathrm{Ph}\right.$ ), 365 (dt, J=2 $2 / 97 \mathrm{~Hz}, 1 \mathrm{H}, \mathrm{NCH}_{2}$ ), 443 (dd, $\mathrm{J}=37 / 99 \mathrm{~Hz}, 1 \mathrm{H}, \mathrm{CHOH}), 450-458(\mathrm{~m}, 1 \mathrm{H}, \mathrm{NCH}), 513$ (t, J=7 7Hz, 1H, OCHCO), 7.2-7 37 (m, 10H,Ph), $\mathrm{OH}$ nicht lokalisiert

b) Nach AAV 2 aus $42 \mathrm{mg}(01 \mathrm{mmol})(R)-1$.

c) Nach AAV 3 aus $42 \mathrm{mg}(0.1 \mathrm{mmol})(R)-1 \mathrm{~b}$.

HPLC-Analyse (n-Hexan/EtOAC = 3/1, 2ml/min), $(R, S)-4 b(91 \mathrm{mn}) /(R, R)-3 \mathrm{~b}(196 \mathrm{mn})$. a) 41/59, b) 15/85, c) $97 / 3$

$\leq(S)-1-B e n z y l-2-f(R)-2-[(R)-2-(4-c h l o r p h e n y l)-2-h y d r o x y-e t h y l l-p y r r o l i d i n o l-2-o x o e t h y l>-2,2-d i m e t h y l-$ proplonat $((R, R)-3 \mathrm{c})$ und

$\leq(S)-1-B e n z y l-2-\{(R)-2-1(S)-2-(4-c h l o r p h e n y l)-2-h y d r o x y-e t h y l l-p y r r o l i d i n o\}-2-o x o e t h y l>-2,2-d i m e t h y l-$ proplonat $(\boldsymbol{R}, S)-4 \mathrm{c})$

a) Nach AAV 1 aus $910 \mathrm{mg}(2 \mathrm{mmol})(R)-1 \mathrm{c}^{14} \mathrm{SC} \mathrm{n}$-Hexan/EtOAC $=1 / 1$.

$(R, R)-3 c$ Farblose Knstalle, Schmp $134^{\circ} \mathrm{C},[\alpha]_{546}=+1510^{\circ},[\alpha]_{578}=+140.7^{\circ}\left(\mathrm{c}=2225, \mathrm{CHCl}_{3}\right)$, Ausb 359

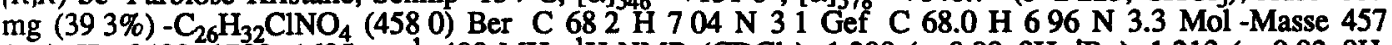
(ms).-IR. 3400,1730, $1635 \mathrm{~cm}^{-1}-400 \mathrm{MHz}^{-1} \mathrm{H}-\mathrm{NMR}\left(\mathrm{CDCl}_{3}\right) .1 .208$ (s, 008x9H, 'Bu), 1213 (s, 0 92x9H, 'Bu), 1 6-1.95 (m, 4H, NCH $\mathrm{CH}_{2} \mathrm{CH}_{2}$ ), 169 (ddd, J=2 6/5.9/14 7Hz, 1H, CH $\left.106 / 147 \mathrm{~Hz}, 1 \mathrm{H}, \mathrm{CH}_{2} \mathrm{C} \mathrm{OH}\right), 273$ (dt, J=7.0/9.5Hz, $\left.0.92 \times 1 \mathrm{H}, \mathrm{NCH}_{2}\right), 309-3.17\left(\mathrm{~m}, 2 \mathrm{H}, \mathrm{CH}_{2} \mathrm{Ph}\right), 3.35-3.42$ $\left(\mathrm{m}, 008 \times 1 \mathrm{H}, \mathrm{NCH}_{2}\right), 354-36\left(\mathrm{~m}, 008 \times 1 \mathrm{H}, \mathrm{NCH}_{2}\right), 364\left(\mathrm{dt}, \mathrm{J}=2 / 9.5 \mathrm{~Hz}, 092 \times 1 \mathrm{H}, \mathrm{NCH}_{2}\right), 4.20-4.27(\mathrm{~m}, 1 \mathrm{H}$, $\mathrm{NCH}), 46-465(\mathrm{~m}, 0$ 08x1H, CHOH), 4.71 (dd, J=2 6/10 6Hz, 092x1H, CHOH), $508(\mathrm{t}, \mathrm{J}=76 \mathrm{~Hz}, 092 \times 1 \mathrm{H}$, OCHCO), 540 (dd, J=6/8Hz, 008x1H, OCHCO), $720-733\left(\mathrm{~m}, 9 \mathrm{H}, \overline{\mathrm{Ph}}, \mathrm{C}_{6} \mathrm{H}_{4}\right), \mathrm{OH}$ nicht lokalisiert Rotamerenverhàltnis: ca. 12/1

$(R, S)-4 \mathrm{c}$ Farblose Knstalle, Schmp $85-87^{\circ} \mathrm{C},[\alpha]_{546}=+953^{\circ},[\alpha]_{578}=+822^{\circ}\left(\mathrm{c}=107, \mathrm{CHCl}_{3}\right)$, Ausb $191 \mathrm{mg}$ (209\%). $-\mathrm{C}_{26} \mathrm{H}_{32} \mathrm{ClNO}_{4}$ (458.0) Ber C $682 \mathrm{H} 704 \mathrm{~N} 31$ Gef. C 68.2 H $714 \mathrm{~N} 30$ Mol.-Masse 457 (ms) -IR $3400,3385,1725,1640,1630 \mathrm{~cm}^{-1}-400 \mathrm{MHz}^{-1} \mathrm{H}-\mathrm{NMR}\left(\mathrm{CDCl}_{3}\right) 121(\mathrm{~s}, 9 \mathrm{H}, \mathrm{tBu}), 148-192(\mathrm{~m}, 6 \mathrm{H}$, $\mathrm{NCH}_{2} \mathrm{CH}_{2} \mathrm{CH}_{2}, \mathrm{CH}_{2} \mathrm{COH}$ ), $276\left(\mathrm{dt}, \mathrm{J}=7.7 / 96 \mathrm{~Hz}, 1 \mathrm{H}, \mathrm{NCH}_{2}\right), 315$ (d, J=7 7Hz, 2H, $\mathrm{CH}_{2} \mathrm{Ph}$ ), 364 (dt, $\left.\mathrm{J}=24 / 96 \mathrm{~Hz}, 1 \mathrm{H}, \mathrm{NCH}_{2}\right), 437-442(\mathrm{~m}, 1 \mathrm{H}, \mathrm{CHOH}), 450(\mathrm{q}, \mathrm{J}=73 \mathrm{~Hz}, 1 \mathrm{H}, \mathrm{NCH}), 510(\mathrm{t}, \mathrm{J}=7 \mathrm{7Hz}, 1 \mathrm{H}$, OCHCO), $553(\mathrm{~d}, \mathrm{~J}=3.7 \mathrm{~Hz}, 1 \mathrm{H}, \mathrm{OH}), 72-737\left(\mathrm{~m}, 9 \mathrm{H}, \mathrm{Ph}, \mathrm{C}_{6} \mathrm{H}_{4}\right)$

b) Nach AAV 2 aus $46 \mathrm{mg}(01 \mathrm{mmol})(R)-1 \mathrm{c}$.

c) Nach AAV 3 aus $456 \mathrm{mg}(1 \mathrm{mmol})(R)-1 \mathrm{c}$, Ausb $304 \mathrm{mg}$ (66 5\%)

HPLC-Analyse (n-Hexan/EtOAc=3/1, 2ml/min), $(R, S)-4 \mathrm{c}(5.3 \mathrm{~min}) /(R, R)-3 \mathrm{c}(131 \mathrm{~min})$ a) $34 / 66$, b) $27 / 73$, c) $92 / 8$

$\leq\langle S\rangle-1$-Benzyl-2-\{(R)-2-I(R)-2-hydroxy-2-(4-methoxyphenyl)-ethyll-pyrrolidino\}-2-oxoethyl>-2,2-dimethylpropionat $((R, R)-3 \mathrm{~d})$ und $\leq(S)-1-\operatorname{Benzy} l-2-\int(R)-2-\int(S)-2-h y d r o x y-2-(4-m e t h o x y p h e n y l)$-ethyll-pyrrolidinol-2-oxoethyl $>-2,2-d i m e t h y l-$ proplonat $((R, S)-4 d)$

1) Nach AAV 1 aus $1353 \mathrm{~g}(3 \mathrm{mmol})(R)-1 \mathrm{~d}^{14} \mathrm{SC} \mathrm{n}-\mathrm{Hexan} / \mathrm{EtOAc}=4 / 6$

$(R, R)-3 \mathrm{~d}$ Farblose Knistalle, Schmp $136^{\circ} \mathrm{C},[\alpha]_{546}=+1658^{\circ},[\alpha]_{578}=+1353^{\circ}\left(\mathrm{c}=14, \mathrm{CHCl}_{3}\right)$, Ausb $553 \mathrm{mg}$ 
(407\%) $-\mathrm{C}_{27} \mathrm{H}_{35} \mathrm{NO}_{5}$ (453.6) Ber. C 71.5 H 7.77 N 3.1 Gef C $71.4 \mathrm{H} 7.83$ N 3.2 Mol.-Masse 453 (ms) -IR $3400,1730,1640 \mathrm{~cm}^{-1} .400 \mathrm{MHz}-{ }^{1} \mathrm{H}-\mathrm{NMR}\left(\mathrm{CDCl}_{3}\right): 1.13\left(\mathrm{~s}, 013 \times 9 \mathrm{H},{ }^{\mathrm{B}} \mathrm{Bu}\right), 1.21$ (s, 0.87x9H, 'Bu), 161-1 88 (m, 4H, $\mathrm{NCH}_{2} \mathrm{CH}_{2} \mathrm{CH}_{2}$ ), 1.67 (ddd, $\mathrm{J}=2.6 / 6.2 / 14.7 \mathrm{~Hz}, 1 \mathrm{H}, \mathrm{CH}_{2} \mathrm{COH}$ ), 2.00 (ddd, J=3 8/ $106 / 147 \mathrm{~Hz}, 1 \mathrm{H}, \mathrm{CH}_{2} \mathrm{COH}, 274\left(\mathrm{dt}, \mathrm{J}=7.0 / 9.5 \mathrm{~Hz}, 087 \times 1 \mathrm{H}, \mathrm{NCH}_{2}\right), 308-3.2\left(\mathrm{~m}, 2 \mathrm{H}, \mathrm{CH}_{2} \mathrm{Ph}\right), 3.35-3.42(\mathrm{~m}$, $\left.013 \times 1 \mathrm{H}, \mathrm{NCH}_{2}\right), 3.54-36\left(\mathrm{~m}, 013 \times 1 \mathrm{H}, \mathrm{NCH}_{2}\right), 3.63\left(\mathrm{dt}, \mathrm{J}=2.2 / 95 \mathrm{~Hz}, 087 \times 1 \mathrm{H}, \mathrm{NCH}_{2}\right), 378(\mathrm{~s}, 087 \times 3 \mathrm{H}$, $\left.\mathrm{OCH}_{3}\right), 380\left(\mathrm{~s}, 013 \times 3 \mathrm{H}, \mathrm{OCH}_{3}\right), 4.20-427(\mathrm{~m}, 0.87 \times 1 \mathrm{H}, \mathrm{NCH}), 4.62$ (dd, J=3/9.5Hz, $0.13 \times 1 \mathrm{H}, \mathrm{CHOH}$ ), 468 (dd, J=2.6/10.6Hz, 0.87x1H, CHOH), $4.70-478(\mathrm{~m}, 013 \times 1 \mathrm{H}, \mathrm{NCH}), 5.12(\mathrm{t}, \mathrm{J}=7.5 \mathrm{~Hz}, 0.87 \times 1 \mathrm{H}$, OCHCO), 543 (dd, J=5/9Hz, $013 \times 1 \bar{H}, O C H C O), 684$ (d, J= 88Hz, 0.87x2H, H-m COHAr), 688 (d, $\mathrm{J}=8 \mathrm{8Hz}, 0$ 13×2H, m-H COHAr), 7.2-7.4 (m, 7H, Ph), OH nicht lokalısiert Rotamerenverhältmis ca. 7/1

$(R, S)-4 \mathrm{~d}$ Farblose Kristalle, Schmp $90-92^{\circ} \mathrm{C},[\alpha]_{546}=+1006^{\circ},[\alpha]_{578}=+81.2^{\circ}\left(\mathrm{c}=165, \mathrm{CHCl}_{3}\right)$, Ausb 402 mg (296\%) - $\mathrm{C}_{27} \mathrm{H}_{35} \mathrm{NO}_{5}$ (453.6) Ber. C $715 \mathrm{H} 7.77$ N 3.1 Gef C 717 H 782 N 3.1 Mol.-Masse 453 (ms) -IR 3400, 1720, $1630 \mathrm{~cm}^{-1} .-400 \mathrm{MHz}^{-1} \mathrm{H}-\mathrm{NMR}\left(\mathrm{CDCl}_{3}\right) 1.22\left(\mathrm{~s}, 9 \mathrm{H},{ }^{\mathrm{B} u}\right), 1.49-1.54(\mathrm{~m}, 1 \mathrm{H}$, $\mathrm{NCH}_{2} \mathrm{CH}_{2} \mathrm{CH}_{2}$ ), 1.61-1.72 (m, 4H, $\mathrm{CH}_{2} \mathrm{COH}, \mathrm{NCH}_{2} \mathrm{CH}_{2} \mathrm{CH}_{2}$ ), 1 82-1 91 (m, 1H, $\mathrm{NCH}_{2} \mathrm{CH}_{2} \mathrm{CH}_{2}$ ), 2.77 (dt, $\left.\mathrm{J}=81 / 96 \mathrm{~Hz}, 1 \mathrm{H}, \mathrm{NCH}_{2}\right), 315\left(\mathrm{~d}, \mathrm{~J}=7.7 \mathrm{~Hz}, 2 \mathrm{H}, \mathrm{CH}_{2} \mathrm{Ph}\right), 3.64\left(\mathrm{dt}, \mathrm{J}=22 / 96 \mathrm{~Hz}, 1 \mathrm{H}, \mathrm{NCH}_{2}\right), 3.78(\mathrm{~s}, 3 \mathrm{H}$, $\left.\mathrm{OCH}_{3}\right), 435-441(\mathrm{~m}, 1 \mathrm{H}, \mathrm{CHOH}), 4.48-456(\mathrm{~m}, 1 \mathrm{H}, \mathrm{NCH}), 512(\mathrm{t}, \mathrm{J}=7.7 \mathrm{~Hz}, 1 \mathrm{H}, \mathrm{OCHCO}), 54(\mathrm{~s}, 1 \mathrm{H}, \mathrm{OH})$ 684 (d, J=8 9Hz, 2H, m-H, COHAr), $724-7.33$ (m, 7H, Ph)

2)

a) Analog zu AAV 3 Umsetzung von $225 \mathrm{mg}(0.05 \mathrm{mmol})(R)-1 \mathrm{~d} \mathrm{mit} 0015 \mathrm{mmol} \mathrm{LiAlH}_{4}$ (1 M-Losung in THF)

b) Nach AAV 2 aus $45 \mathrm{mg}$ (0 $1 \mathrm{mmol})(R)$-1d.

c) wie b) aber in abs Ether

d) wie b) aber be1 $-40^{\circ} \mathrm{C}$, Reaktionsdauer $48 \mathrm{~h}$

e) wie b) aber in abs Toluol

f) Analog zu AAV 3 Umsetzung von $225 \mathrm{mg}(005 \mathrm{mmol})(R)-1 d$ mit $006 \mathrm{mmol} \mathrm{Li}(\mathrm{OMe})_{3} \mathrm{AlH}$ (1M-Losung

in THF, dargestellt aus $1 \mathrm{M}-\mathrm{LiAlH}_{4}$ in THF und 3 Aquivalenten abs $\mathrm{MeOH}$ )

g) wie f) aber bei $-15^{\circ} \mathrm{C}$

h) Analog zu AAV 3 Umsetzung von $45 \mathrm{mg}(01 \mathrm{mmol})$ (R)-1d mit $012 \mathrm{mmol} \mathrm{LiEt} \mathrm{t}_{3} \mathrm{BH}$ (1 M-Los in THF)

1) wie h) aber in abs Toluol

j) Eine Losung von $203 \mu \mathrm{l}(01 \mathrm{mmol}) 15$-crown-5 (298\%) in $05 \mathrm{ml}$ abs THF wurde be1 $-78^{\circ} \mathrm{C}$ unter Ruhren mit 01 mmol LiEt 3 BH (1M-Lösung) versetzt (vgl dazu Lit ${ }^{21}$ ) und nach $10 \mathrm{~min}$ wurden $225 \mathrm{mg}(005 \mathrm{mmol})$ (R)-1d in $025 \mathrm{ml}$ abs THF, zugegeben Aufarbeitung nach $1 \mathrm{~h}$ wie bei AAV 3.

k) Analog zu AAV 3 Umsetzung von $45 \mathrm{mg}(01 \mathrm{mmol})(R)$-1d mit $012 \mathrm{mmol} \mathrm{DiBalH}$ (1 M-Lösung in n-Hexan)

1) Analog zu AAV 3 Umsetzung von $45 \mathrm{mg}(01 \mathrm{mmol})(R)-1 \mathrm{~d}$ mit $012 \mathrm{mmol} \mathrm{Li}\left({ }^{(} \mathrm{Bu}\right)_{3} \mathrm{BH}(1 \mathrm{M}-\mathrm{Losung}$ in THF)

m) wie 1) aber in abs Toluol

n) Nach AAV 3 aus $451 \mathrm{mg}(1 \mathrm{mmol})(R)$-1d Umknstallisation aus Dusopropylether lieferte $(R, S)$-4d Ausb $285 \mathrm{mg}(63 \%)$

o) Analog zu AAV 3 Umsetzung von $45 \mathrm{mg}(01 \mathrm{mmol})(R)-1 \mathrm{~d}$ in Toluol

HPLC-Analyse (n-Hexan/EtOAc= $7 / 3,2 \mathrm{ml} / \mathrm{min}),(R, S)-4 \mathrm{~d}(80 \mathrm{~mm}) /(R, R)-3 \mathrm{~d}(164 \mathrm{~min})$ a) $27 / 73$, b) $22 / 78$, c) $22 / 78$, d) $40 / 60$, e) $26 / 74$, f) $24 / 76$, g) $21 / 79$, h) $32 / 68$, i) $24 / 76$, j) $40 / 60$, k) $76 / 24$, l) $93 / 7$, m) $86 / 14$, n) $98 / 2$, o) $97 / 3$

$\leq(S)-1-B e n z y l-2-\left\{(R)-2-\left[(R)-2-\left(4^{t}\right.\right.\right.$ butyldimethylszlyloxy-3-methoxyphenyl $\left.)-2-h y d r o x y e t h y l l-p y r r o l i d i n o\right\}-2-$ oxoethyl $>-2.2$-dimethylpropionat $((R, R)-3$ e) und

$\leq(S)-1-$ Benzyl-2-f(R)-2-[(S)-2-(4-butyldımethylsilyloxy-3-methoxyphenyl)-2-hydroxyethyl - -pyrrolldinol-2oxoethyl>-2.2-dimethylpropionat $((R, S)-4 e)$

a) Nach AAV 2 aus $(R)-1 \mathrm{e}^{14}$, in Lit ${ }^{14}$ beschrieben

b) Nach AAV 1 aus $465 \mathrm{mg}(08 \mathrm{mmol})(R)$-1e SC n-Hexan/EtOAC =1/1 (R,R)-3e Ausb $215 \mathrm{mg}(462 \%)$

$(R, S)-4 \mathrm{e}$ Ausb $80 \mathrm{mg}(17.2 \%)$

c) Nach AAV 3 aus $58 \mathrm{mg}(01 \mathrm{mmol})(R)-1 \mathrm{e}$.

HPLC-Analyse (n-Hexan/EtOAc=7/3), $(R, S)-4$ e $(64 \mathrm{~min}) /(R, R)-3 e(117 \mathrm{~min})$ a) $14 / 86, \mathrm{~b}) 37 / 63, \mathrm{c}) 96 / 4$

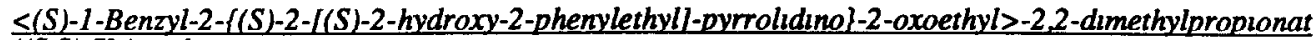

$((S, S)-5 b)$ und

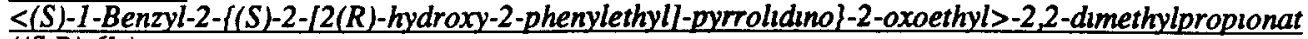

$((S, R)-6 \mathbf{b})$

Nach AAV 1 aus $842 \mathrm{mg}(2 \mathrm{mmol})(S)-2 b^{14} \mathrm{SC}$. $\mathrm{n}-\mathrm{Hexan} / \mathrm{EtOAc}=7 / 3$

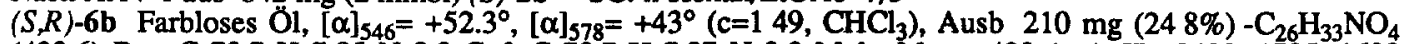
(4236) Ber C 737 H 785 N 33 Gef C 737 H 797 N 33 Mol - Masse 423 (ms) -IR 3400,1725 , 1630 $\mathrm{cm}^{-1}-400 \mathrm{MHz}^{-1} \mathrm{H}-\mathrm{NMR}\left(\mathrm{CDCl}_{3}\right) 119\left(\mathrm{~s}, 008 \times 9 \mathrm{H},{ }^{\mathrm{B} u}\right), 121(\mathrm{~s}, 092 \times 9 \mathrm{H}, \mathrm{Bu}), 156-202(\mathrm{~m}, 6 \mathrm{H}$, 
$\left.\mathrm{NCH}_{2} \mathrm{CH}_{2} \mathrm{CH}_{2}, \mathrm{CH}_{2} \mathrm{COH}\right), 2.93-3.00(\mathrm{~m}, 0.08 \times 2 \mathrm{H}), 3.10-3.25\left(0.92 \times 3 \mathrm{H}, \mathrm{NCH}_{2}, \mathrm{CH}_{2} \mathrm{Ph}\right), 338-3.45(\mathrm{~m}$,

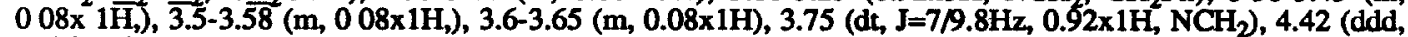
$\mathrm{J}=4$ 0/5.6/.6Hz, $092 \times 1 \mathrm{H}, \mathrm{CHOH}), 455-4.65(\mathrm{~m}, 092 \times 1 \mathrm{H}, \mathrm{NCH}), 4.69-478(\mathrm{~m}, 008 \times 1 \mathrm{H}, \mathrm{CHOH}), 512(\mathrm{~d}$, $\mathrm{J}=4 \mathrm{OHz}, 1 \mathrm{H}, \mathrm{OH}), 5.22(\mathrm{dd}, \mathrm{J}=7.8 / 6.7 \mathrm{~Hz}, 092 \times 1 \mathrm{H}, \mathrm{OCHCO}), 555(\mathrm{t}, \mathrm{J}=72 \mathrm{~Hz}, 0.08 \times 1 \mathrm{H}, \mathrm{OCHCO}), 72-738$ (m, 10H, Ph), Rotamerenverhälmis' ca. 11/1

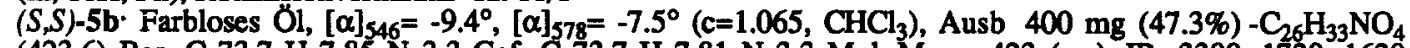
(423 6) Ber. C 73.7 H 7.85 N 3.3 Gef. C 73.7 H 7.81 N 3.3 Mol.-Masse 423 (ms) -IR: $3300,1720,1620$ $\mathrm{cm}^{-1}-400 \mathrm{MHz}-{ }^{1} \mathrm{H}-\mathrm{NMR}\left(\mathrm{CDCl}_{3}\right): 1.19\left(\mathrm{~s}, 0.2 \times 9 \mathrm{H}, \mathrm{t}_{\mathrm{Bu}}\right), 1.20\left(\mathrm{~s}, 08 \times 9 \mathrm{H},{ }_{\mathrm{Bu}}\right), 16-205(\mathrm{~m}, 4 \mathrm{H}$, $\mathrm{NCH}_{2} \mathrm{CH}_{2} \mathrm{CH}_{2}$ ), 1.63 (ddd, $\mathrm{J}=2.9 / 6.2 / 145 \mathrm{~Hz}, 1 \mathrm{H}, \mathrm{CH}_{2} \mathrm{COH}$ ), 1.98 (ddd, $\mathrm{J}=4.8 / 10 / 14.5 \mathrm{~Hz}, 1 \mathrm{H}, \mathrm{CH}_{2} \mathrm{COH}$ ), $232-24(\mathrm{~m}, 02 \times 2 \mathrm{H}), 2.95-312\left(\mathrm{~m}, 0.8 \times 3 \mathrm{H}, \mathrm{NCH}_{2}, \mathrm{CH}_{2} \mathrm{Ph}\right), 337-3.42\left(\mathrm{~m}, 02 \times 1 \mathrm{H}, \mathrm{NCH}_{2}\right), 345-352(\mathrm{~m}$, $\left.02 \times 1 \mathrm{H}, \mathrm{NCH}_{2}\right), 362\left(\mathrm{dt}, \mathrm{J}=73 / 9.3 \mathrm{~Hz}, 0.8 \times 1 \mathrm{H}, \mathrm{NCH}_{2}\right), 3.9-396(\mathrm{~m}, 0.2 \times 1 \mathrm{H}, \mathrm{NCH}), 4.35-4.40(\mathrm{~m}, 08 \times 1 \mathrm{H}$, $\mathrm{NCH}$ ), 459 (dd, J=2/ 10Hz, 0.2x1H, CHOH), 474 (dd, $\mathrm{J}=29 / 10 \mathrm{~Hz}, 0.8 \times 1 \mathrm{H}, \mathrm{CHOH}$ ), 506 (dd, J=6.9/7.9Hz, $08 \times 1 \mathrm{H}, \mathrm{OCHCO}), 5.46(\mathrm{t}, \mathrm{J}=73 \mathrm{~Hz}, 02 \times 1 \mathrm{H}, \mathrm{OCHCO}), 7.2-74(\mathrm{~m}, 10 \mathrm{H}, \mathrm{Ph}), \mathrm{OH}$ nicht lokalisiert Rotamerenverhältmis ca. $4 / 1$

b) Nach AAV 2 aus $42 \mathrm{mg}(01 \mathrm{mmol})(S-2 \mathrm{~b}$.

c) Nach AAV 3 aus $42 \mathrm{mg}(01 \mathrm{mmol})(S)-2 \mathrm{~b}$.

HPLC-Analyse (n-Hexan/EtOAc=7/3, 2ml/min, $\left.50^{\circ} \mathrm{C}\right),(S, R)-6 \mathrm{~b}(5.2 \mathrm{~min}) /(S, S)-5 \mathrm{~b}(10.5 \mathrm{~min})$ a) $\left.36 / 64, \mathrm{~b}\right)$ $2 / 98$ c' $5 / 2$ nach $400 \mathrm{MHz}^{-1} \mathrm{H}-\mathrm{NMR}$

$\leq(S)-1-B e n z y l-2-\{(S)-2-[(S)-2-(4-c h l o r p h e n y l)-2-h y d r o x y-e t h y l l-p y r r o l z d i n o l-2-o x o e t h y l>-2,2-d i m e t h y l-$ proplonat $((S, S)-5 \mathbf{c})$ und

$\leq(S)-1-B e n z y l-2-(S)-2-(R)-2-(4-c h l o r p h e n y l)-2-h y d r o x y-e t h y l l-p y r r o l i d i n o\}-2-o x o e t h y l>-2,2-d i m e t h y l-$ proplonat $((S, R)-6 \mathrm{c})$

Nach AAV 1 aus $600 \mathrm{mg}$ (1 $32 \mathrm{mmol})(S)$-2 $\mathrm{c}^{14}$ Trennung durch MPLC: $\mathrm{n}$-Hexan/EtOAc=1/1 $(S, R)-6 \mathrm{c}$ wurde zusatzlich durch prap HPLC ( $\mathrm{n}-\mathrm{Hexan} / \mathrm{E} \mathrm{OAc}=1 / 1,12 \mathrm{ml} / \mathrm{min}$,) gereinigt.

$(S, R)$-6c. Farblose Kristalle, Schmp $171^{\circ} \mathrm{C}[\alpha]_{546}=+558^{\circ},[\alpha]_{578}=+465^{\circ}\left(\mathrm{c}=043, \mathrm{CHCl}_{3}\right.$ ), Ausb $145 \mathrm{mg}$ $(240 \%)-\mathrm{C}_{26} \mathrm{H}_{32} \mathrm{ClNO}_{4}(4580)$ Ber C $68.2 \mathrm{H} 704 \mathrm{~N} 31 \mathrm{Gef}$ C $682 \mathrm{H} 709 \mathrm{~N} 31 \mathrm{Mol}-\mathrm{Masse} 457$ (ms) -IR 3350, 1720, $1630 \mathrm{~cm}^{-1}-400 \mathrm{MHz}^{1} \mathrm{H}-\mathrm{NMR}\left(\mathrm{CDCl}_{3}\right) 118\left(\mathrm{~s}, 006 \times 9 \mathrm{H}, \mathrm{t}^{\mathrm{Bu}}\right), 121\left(\mathrm{~s}, 094 \times 9 \mathrm{H}, \mathrm{t}^{\mathrm{B} u}\right)$, $155-162\left(\mathrm{~m}, 3 \mathrm{H}, \mathrm{NCH}_{2} \mathrm{CH}_{2} \mathrm{CH}_{2}, \mathrm{CH}_{2} \mathrm{CHOH}\right), 185-20\left(\mathrm{~m}, 3 \mathrm{H}, \mathrm{NCH}_{2} \mathrm{CH}_{2} \mathrm{CH}_{2}\right), 2.95-3.07$ (m, $\left.006 \times 2 \mathrm{H}\right)$, $311-325\left(\mathrm{~m}, 0.94 \times 3 \mathrm{H}, \mathrm{NCH}_{2}, \mathrm{CH}_{2} \mathrm{Ph}\right), 3.35-3.43(\mathrm{~m}, 0.06 \times 1 \mathrm{H}), 3.53-358(\mathrm{~m}, 0.06 \times 1 \mathrm{H}), 362-366(\mathrm{~m}$, $006 \times 1 \mathrm{H}), 372-3.79\left(\mathrm{~m}, 0.94 \times 1 \mathrm{H}, \mathrm{NCH}_{2}\right), 430-436(\mathrm{~m}, 0.94 \times 1 \mathrm{H}, \mathrm{CHOH}), 452-4.59(\mathrm{~m}, 094 \times 1 \mathrm{H}, \mathrm{NCH})$, $472-477$ (m, 0.06x1H, CHOH), 522 (dd, J=6 $7 / 7$ 8Hz, 1H, OCHCO), 526 (d, J=4 OHz, 1H,OH), $723-733$ (m, 9H, Ph, $\mathrm{C}_{6} \mathrm{H}_{4}$ ), Rotamerenverhältnis ca. $15 / 1$

$(S, S)-5 \mathrm{c}$ Farblose Kristalle, Schmp $99-100^{\circ} \mathrm{C},[\alpha]_{546}=-98^{\circ},[\alpha]_{578}=-8.7^{\circ}\left(\mathrm{c}=0915, \mathrm{CHCl}_{3}\right)$, Ausb $275 \mathrm{mg}$ (456\%) $-\mathrm{C}_{26} \mathrm{H}_{32} \mathrm{ClNO}_{4}(4580)$ Ber C $682 \mathrm{H} 704 \mathrm{~N} 3.1 \mathrm{Gef} \mathrm{C} 682 \mathrm{H} 710 \mathrm{~N} 31 \mathrm{Mol}$-Masse 457 (ms) -IR $3400,1720,1660,1645 \mathrm{~cm}^{-1}-400 \mathrm{MHz}^{1} \mathrm{H}-\mathrm{NMR}\left(\mathrm{CDCl}_{3}\right) 119\left(\mathrm{~s}, 013 \times 9 \mathrm{H},{ }^{\mathrm{t}} \mathrm{Bu}\right), 120$ (s, $\left.087 \times 9 \mathrm{H}, \mathrm{Bu}\right)$, $152-207$ (m, 4H, $\mathrm{NCH}_{2} \mathrm{CH}_{2} \mathrm{CH}_{2}$ ) 157 (ddd, J=2 8/6 1/14 5Hz, $1 \mathrm{H}, \mathrm{CH}_{2} \mathrm{COH}$ ), 190 (ddd,

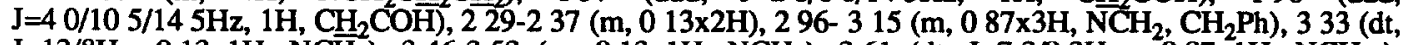

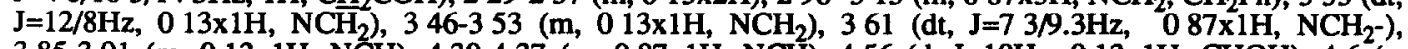
$385-391$ (m, $013 \times 1 \mathrm{H}, \mathrm{NCH}), 430-4.37$ (m, 0.87x1H, NCH), 456 (d, J=10Hz, $013 \times 1 \mathrm{H}, \mathrm{CHOH}), 46$ (s, breit, $1 \mathrm{H}, \mathrm{OH}), 4.72$ (dd, J=2 8/10 5Hz, $087 \times 1 \mathrm{H}, \mathrm{CHOH}), 507$ (dd, J=6 $9 / 78 \mathrm{~Hz}, 087 \times 1 \mathrm{H}, \mathrm{OCHCO}), 543$ (t, $\mathrm{J}=7 \mathrm{~Hz}, 013 \times 1 \mathrm{H}, \mathrm{OCHCO}), 719-734\left(\mathrm{~m}, 9 \mathrm{H}, \mathrm{Ph}, \mathrm{C}_{6} \mathrm{H}_{4}\right)$, Rotamerenverhaltn1s ca $7 / 1$

b) Nach AAV 2 aus $46 \mathrm{mg}(01 \mathrm{mmol})(S)$-2c.

c) Nach AAV 3 aus $46 \mathrm{mg}(01 \mathrm{mmol})(S)$-2c.

Isomerenverhältnisse

a) prap HPLC (n-Hexan/EtOAc=1/1, $12 \mathrm{ml} / \mathrm{min}),(S, R)-6 \mathrm{c}(9.0 \mathrm{mun}) /(S, S)-5 \mathrm{c}(144 \mathrm{~min}) \cdot 42 / 58$

b) HPLC-Analyse (n-Hexan/EtOAc=7/3,2ml/min), $(S, R)-6 \mathrm{c}(47 \mathrm{~min}) /(S, S)-5 \mathrm{c}(120 \mathrm{~min})>5 / 95$

c) $(S, R)-6 \mathrm{c} /(S, S)-5 \mathrm{c} \cdot 5 / 2$ nach $400 \mathrm{MHz}-{ }_{\mathrm{H}} \mathrm{H}-\mathrm{NMR}$

$\leq(S)-1-B e n z y l-2-((S)-2-[(S)-2-h y d r o x y-2-(4-m e t h o x y p h e n y l)-e t h y l l-p y r r o l l d i n o l-2-o x o e t h y l>-2,2-d i m e t h y l-$ proplonat $((\boldsymbol{S}, S)-5 \mathrm{~d})$ und

$\left\langle(S)-1-B e n z y l-2-\int(S)-2-1(R)-2-h y d r o x y-2-(4-m e t h o x y p h e n y l)\right.$-ethyll-pyrrolldinol-2-oxoethyl $>-2,2-d i m e t h y l-$ proplonat $((S, R)-6 \mathrm{~d})$

Nach AAV 1 aus $927 \mathrm{mg}(206 \mathrm{mmol})(S)-2 \mathrm{~d}^{14}$ Trennung durch MPLC $\mathrm{n}-\mathrm{Hexan} / \mathrm{EtOAC}=1 / 1$

$(S, R)$-6d Farblose Kristalle, Schmp $113-115^{\circ} \mathrm{C},[\alpha]_{546}=+496^{\circ},[\alpha]_{578}=+393^{\circ}\left(\mathrm{c}=13, \mathrm{CHCl}_{3}\right)$, Ausb 220 $\mathrm{mg}(236 \%)-\mathrm{C}_{27} \mathrm{H}_{35} \mathrm{NO}_{5}(4536) \mathrm{Ber} \mathrm{C} 715 \mathrm{H} 778 \mathrm{~N} 31$ Gef C $715 \mathrm{H} 775 \mathrm{~N} 3.0 \mathrm{Mol}$-Masse 453 (ms) -IR 3370, 1720, $1630 \mathrm{~cm}^{-1}-400 \mathrm{MHz}^{1}{ }^{\mathrm{H}} \mathrm{H}-\mathrm{NMR}\left(\mathrm{CDCl}_{3}\right) 119$ (s, 008x9H, 'Bu), 120 (s, 092x9H, 'Bu), $157-199\left(\mathrm{~m}, 6 \mathrm{H}, \mathrm{NCH}_{2} \mathrm{CH}_{2} \mathrm{CH}_{2}, \mathrm{CH}_{2} \mathrm{COH}\right), 292-303(\mathrm{~m}, 0.08 \times 2 \mathrm{H}), 311-3.25\left(\mathrm{~m}, 092 \times 3 \mathrm{H}, \mathrm{NCH}_{2}\right.$, $\mathrm{CH}_{2} \mathrm{Ph}$ ), 34 (ddd, $\left.\mathrm{J}=3 / 8 / 13 \mathrm{~Hz}, 008 \times 1 \mathrm{H},\right), 352(\mathrm{dt}, \mathrm{J}=2 / 13 \mathrm{~Hz}, 0.08 \times 1 \mathrm{H}), 362-367(\mathrm{~m}, 008 \times 1 \mathrm{H}), 370-378$ $\left(\mathrm{m}, 092 \times 1 \mathrm{H}, \mathrm{NCH}_{2}\right), 379(\mathrm{~s}, 092 \times 3 \mathrm{H}, \mathrm{OMe}), 381(\mathrm{~s}, 008 \times 3 \mathrm{H}, \mathrm{OMe}), 437$ (dd, J=4 0/9 3Hz, $092 \times 1 \mathrm{H}$, CHOH), $453-460$ (m, 0 92x1H, NCH-), 468 (dd, J=4/10Hz, 0 08x1H, CHOH), 505 (s, breit, 1H, OH), 520 (dd, J=6 $7 / 79 \mathrm{~Hz}, 0.92 \times 1 \mathrm{H}, \mathrm{OCHCO}), 548$ (dd, J=7/8Hz, $008 \mathrm{Hx} 1 \mathrm{H}, \mathrm{OCHCO}), 686\left(\mathrm{~d}, \mathrm{~J}=89 \mathrm{~Hz}, 2 \mathrm{H}, \mathrm{C}_{6} \mathrm{H}_{4}\right.$ ), 
7 20-7 32 (m, 7H, $\mathrm{Ph}_{3} \mathrm{C}_{6} \mathrm{H}_{4}$ ), Rotamerenverhältmis: ca.12/1.

$(S, S)$-5d Farbloses Ol, $[\alpha]_{546}=-10.3^{\circ},[\alpha]_{578}=-9.0^{\circ}\left(\mathrm{c}=225, \mathrm{CHCl}_{3}\right)$, Ausb. $385 \mathrm{mg}(414 \%)-\mathrm{C}_{27} \mathrm{H}_{35} \mathrm{NO}_{5}$ (453 6) Ber. C 715 H 7.78 N 31 Gef C 712 H 798 N 3.0 Mol.Masse 453 (ms)-IR: $3400,1720,1660$ $\mathrm{cm}^{-1}-400 \mathrm{MHz}^{1} \mathrm{H}-\mathrm{NMR}\left(\mathrm{CDCl}_{3}\right): 1.19$ (s, 0 2x9H, $\left.\mathrm{Bu}\right), 1.20$ (s, $\left.08 \times 9 \mathrm{H}, \mathrm{Bu}\right), 155-2.05(\mathrm{~m}, 4 \mathrm{H}$, $\mathrm{NCH}_{2} \mathrm{CH}_{2} \mathrm{CH}_{2}$ ), 1.58 (ddd, $\mathrm{J}=3 / 6.5 / 14.5 \mathrm{~Hz}, 1 \mathrm{H}, \mathrm{CH}_{2} \mathrm{COH}$ ), 2.00 (ddd, $\mathrm{J}=4.9 / 10 / 145 \mathrm{~Hz}, 1 \mathrm{H}, \mathrm{CH}_{2} \mathrm{COH}$ ), $236-244(\mathrm{~m}, 0.2 \times 2 \mathrm{H}), 2.97-3.15\left(\mathrm{~m}, 0.8 \times 3 \mathrm{H}, \mathrm{NCH}_{2}, \mathrm{CH}_{2} \mathrm{Ph}\right), 334\left(\mathrm{dt}, \mathrm{J}=12 / 9 \mathrm{~Hz}, 0.2 \times 1 \mathrm{H}, \mathrm{NCH}_{2}\right), 347-354$ $\left(\mathrm{m}, 02 \times 1 \mathrm{H}, \mathrm{NCH}_{2}\right), 3.61$ (dt, J=7.3/9.7Hz, $08 \times 1 \mathrm{H}, \mathrm{NCH}_{2}$ ), 3.79 (s, $\left.0.8 \times 3 \mathrm{H}, \mathrm{OMe}\right), 3.80$ (s, 0.2x3H, OMe), $392-398(\mathrm{~m}, 0.2 \times 1 \mathrm{H}, \mathrm{NCH}), 4.32-4.38(\mathrm{~m}, 0.8 \times 1 \mathrm{H}, \mathrm{NCH}), 453$ (d, J=9Hz, 0.2x1H, CHOH), 4.70 (dd, $\mathrm{J}=3 / 10 \mathrm{~Hz}, 08 \times 1 \mathrm{H}, \mathrm{CHOH}$ ), 5.07 (dd, J=6.8/8.0Hz, 0.8x1H, OCHCO), 5.47 (t, J=7 5Hz, $02 \times 1 \mathrm{H}, \mathrm{OCHCO}$ ), $688\left(\mathrm{~d}, \mathrm{~J}=8 \mathrm{Hzz}, 2 \mathrm{H}, \mathrm{C}_{6} \mathrm{H}_{4}\right), 7.19-7.32\left(\mathrm{~m}, 7 \mathrm{H}, \mathrm{Ph}, \mathrm{C}_{6} \mathrm{H}_{4}\right)$, Rotamerenverhalltnis. ca $4 / 1$.

b) Nach AAV 2 aus $46 \mathrm{mg}(0.1 \mathrm{mmol})$ (S)-2d.

c) Nach AAV 3 aus $46 \mathrm{mg}(01 \mathrm{mmol})(S)-2 \mathrm{~d}$.

HPLC-Analyse (n-Hexan/EtOAc=7/3, 2ml/min); (S,R)-6d $(80 \mathrm{~min}) /(S, S)-5 \mathrm{~d}(164 \mathrm{~min})$ a) $35 / 65 \mathrm{~b}) 5 / 95 \mathrm{c})$ 4/1 nach $400 \mathrm{MHz}^{-1} \mathrm{H}-\mathrm{NMR}$

N-(2,2-Dimethylpropionyl)-(S)-pyrrolidin-2-carbonsäure ((S)-7)

Die Substanz wurde als Nebenprodukt bei der Darstellung von N-[(S)-2-(2,2-Dimethylpropionyloxy)-3phenylpropionyl]-(S)-pyrrolıdın-2-carbonsáure ${ }^{26}$ 1soliert Farblose Knstalle, Schmp 124-127 ${ }^{\circ}$, (Lit. ${ }^{27} 1283$ $-126^{\circ} \mathrm{C}$ ), Ausb $209 \mathrm{~g}(21 \%)$

\section{1-(2-Methoxy-1-pyrrolidinyl)-2,2-dimethylpropanon ((rac)-8)}

Darstellung aus $1.6 \mathrm{~g}(80 \mathrm{mmol})(S)-7$ durch elektrochemische Oxidation (in abs $\mathrm{MeOH} / \mathrm{NaOMe}$ ) analog zu dem in Lit ${ }^{26}$ angegebenen Verfahren (300 mA, ca. $18 \mathrm{~V}$ ) SC-Reinigung mit $\mathrm{n}$-Hexan/EtOAc/Dimethylethylamin $=90 / 8 / 2$

Farbloses Òl, Ausb $961 \mathrm{mg}(65 \%)-\mathrm{C}_{10} \mathrm{H}_{19} \mathrm{NO}_{2}(1853)$ Ber $\mathrm{C} 64.8 \mathrm{H} 1034 \mathrm{~N} 76$ Gef C $652 \mathrm{H} 970 \mathrm{~N} 79$ Mol Masse 185 (ms) -IR $1660 \mathrm{~cm}^{-1}-60 \mathrm{MHz}^{-1} \mathrm{H}-\mathrm{NMR}\left(\mathrm{CDCl}_{3}\right)^{\cdot} 125(\mathrm{~s}, 9 \mathrm{H}, \mathrm{Bu}), 16-22(\mathrm{~m}, 4 \mathrm{H}$, $\left.\mathrm{NCH}_{2} \mathrm{CH}_{2} \mathrm{CH}_{2}\right), 33(\mathrm{~s}, 3 \mathrm{H}, \mathrm{OMe}), 345-385\left(\mathrm{~m}, 2 \mathrm{H}, \mathrm{NCH}_{2}\right), 55(\mathrm{~d}, \mathrm{~J}=4 \mathrm{OHz}, 1 \mathrm{H}, \mathrm{NCHO})$

\section{1-12-(2-Oxo-2-phenylethyl)-1-pyrrolidinyll-2,2-dimethylpropanon ((rac)-9)}

Darstellung analog zu dem in Lit ${ }^{25}$ angegebenen Verfahren aus $400 \mathrm{mg}(216 \mathrm{mmol})(\mathrm{rac})-8$ und $622 \mathrm{mg}$ (3 $24 \mathrm{mmol}$ ) 1-Phenyl-1-trimethylsilyloxyethen

Farblose Knstalle, Schmp $80-82^{\circ} \mathrm{C}$, Ausb $330 \mathrm{mg}(56 \%)-\mathrm{C}_{17} \mathrm{H}_{23} \mathrm{NO}_{2}(2734)$ Ber C $747 \mathrm{H} 848 \mathrm{~N} 51 \mathrm{Gef}$ C 748 H 843 N 51 Mol -Masse 273 (ms) -IR $1680,1610 \mathrm{~cm}^{-1}-400 \mathrm{MHz}-{ }^{1} \mathrm{H}-\mathrm{NMR}\left(-20^{\circ} \mathrm{C}, \mathrm{CDCl}_{3}\right): 129$ (s, $9 \mathrm{H},{ }^{\mathrm{t}} \mathrm{Bu}$ ), 1.71-1 78 (m, 1H, NCH $\left.\mathrm{CH}_{2} \mathrm{CH}_{2}\right), 185-206$ (m, 3H, $\mathrm{NCH}_{2} \mathrm{CH}_{2} \mathrm{CH}_{2}$ ), 2.64 (dd, J=10 3/14.3Hz, $1 \mathrm{H}$, $\mathrm{CH}_{2} \mathrm{CO}$ ), $360-374\left(\mathrm{~m}, 2 \mathrm{H}, \mathrm{NCH}_{2}\right), 394$ (dd, J=2 9/143Hz, 1H, $\left.\mathrm{CH}_{2} \mathrm{CO}\right), 457-463(\mathrm{~m}, 1 \mathrm{H}, \mathrm{NCH})$, 7 48-7 61 (m, 3H, H-m/p-Ph), 814 (d, J=7 3Hz, 2H, H-o-Ph)

\section{1-[(R,S)-2-((R,S)-2-Hydroxy-2-phenylethyl)-1-pyrrolidinyll-2,2-dimethylpropanon ((rac)-10)}

I- $((R, S)-2-((S, R)-2-H y d r o x y-2-p h e n y l e t h y l)-1$-pyrrolidinyll-2,2-dimethylpropanon ((rac)-11)

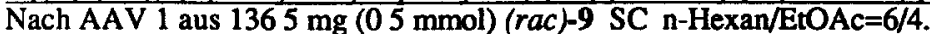

(rac)-11 Farblose Knstalle, Schmp 101-102 ${ }^{\circ} \mathrm{C}$, Aus $46 \mathrm{mg}(335 \%) \cdot-\mathrm{C}_{17} \mathrm{H}_{25} \mathrm{NO}_{2}$ (275.5) Ber C $741 \mathrm{H} 915$ N 51 Gef. C $741 \mathrm{H} 935 \mathrm{~N} 51 \mathrm{Mol}$-Masse 275 (ms) -IR: $3360,1595 \mathrm{~cm}^{-1}-400 \mathrm{MHz}^{1} \mathrm{H}-\mathrm{NMR}\left(\mathrm{CDCl}_{3}\right)$ $127\left(\mathrm{~s}, 9 \mathrm{H},-{ }^{\mathrm{t}} \mathrm{Bu}\right), 1.48-204\left(\mathrm{~m}, 6 \mathrm{H},-\mathrm{N}-\mathrm{CH}_{2}-\mathrm{CH}_{2}-\mathrm{CH}_{2},-\mathrm{CH}_{2} \mathrm{C}-\mathrm{OH}\right), 359(\mathrm{~m}, \mathrm{~J}=46 / 77 / 108 \mathrm{~Hz}, 1 \mathrm{H}$, $\left.-\mathrm{N}-\mathrm{CH}_{2}\right), 380\left(\mathrm{dt}, \mathrm{J}=10.8 / 7.7 \mathrm{~Hz}, 1 \mathrm{H},-\mathrm{N}-\mathrm{CH}_{2}\right), 4 \overline{49}$ (dt, J=4 2/8 8Hz, $\left.1 \mathrm{H},-\mathrm{CH}-\mathrm{OH}\right), 462-4.68(\mathrm{~m}, 1 \mathrm{H}$, -N-CH), 541 (s, 1H, -OH), 7 20-7 $38(\mathrm{~m}, 5 \mathrm{H},-\mathrm{Ph})$.

(rac )-10 Farblose Kristalle, Schmp 82-83 ${ }^{\circ} \mathrm{C}$, Ausbeute $41 \mathrm{mg}(298 \%)-\mathrm{C}_{17} \mathrm{H}_{25} \mathrm{NO}_{2}$ (275 5) Ber C $741 \mathrm{H}$ $915 \mathrm{~N} 51 \mathrm{Gef}$ C $741 \mathrm{H} 921 \mathrm{~N} 5.1 \mathrm{Mol}$-Masse $275(\mathrm{~ms})-\mathrm{IR} 3400,1600, \mathrm{~cm}^{-1}-400 \mathrm{MHz}-{ }^{-1} \mathrm{H}-\mathrm{NMR}$ $\left(\mathrm{CDCl}_{3}\right) 121$ (s,9H,-'Bu), 12205 (m,5H,-N-CH$-\mathrm{CH}_{2}-\mathrm{CH}_{2}-,-\mathrm{CH}_{2} \mathrm{C}-\mathrm{OH}-$ ), 176 (ddd,J=2 6/6 8/14.5Hz, $\left.1 \mathrm{H},-\mathrm{CH}_{2} \mathrm{C}-\mathrm{OH}-\right), 35-365$ (m,2H,-N-CH $\left.2^{-}\right), 438-444$ (m,1H, -N-CH-), 472 (d,J=9 9Hz,1H,-Cㅍ-OH-), $720-738(\mathrm{~m}, 5 \mathrm{H},-\mathrm{Ph}),,-\mathrm{OH}$ nicht lokalisiert

b) $27 \mathrm{mg}(01 \mathrm{mmol})$ ( $\mathrm{rac})-9$ wurden in $1.5 \mathrm{ml}$ THF mit $80 \mathrm{mg}(032 \mathrm{mmol}) \mathrm{Li}\left(\mathrm{O}^{\mathrm{t} B u}\right)_{3} \mathrm{AlH}$ nach AAV 2 reduziert

c) $27 \mathrm{mg}(01 \mathrm{mmol})$ (rac)-9 wurden in $15 \mathrm{ml}$ THF mit 012 mmol $\mathrm{LiEt}_{3} \mathrm{BH}$ be1 $-78^{\circ} \mathrm{C}$ nach AAV 3 reduziert

d) $27 \mathrm{mg}(01 \mathrm{mmol})(\mathrm{rac})-9$ wurden in $15 \mathrm{ml}$ THF mit $012 \mathrm{mmol} \mathrm{K}\left({ }^{\mathrm{s}} \mathrm{Bu}\right)_{3} \mathrm{BH}$ be1 $-78^{\circ} \mathrm{C}$ nach AAV 3 reduziert

HPLC-Analyse (n-Hexan/Ethylacetat=7 3, 2ml/min), ( rac)-10 $(47 \mathrm{~min}) /(\mathrm{rac})-11(115 \mathrm{~min})$ a) 46/54, b) $60 / 40$, c) $45 / 55$, d) $19 / 81$

AAV 4 - Hydrolyse der Amidoalkohole

a) Eine Losung des zu hydrolysierenden Amidoalkohols (1 mmol) in THF (40 ml) wird zusammen mit Tetrabutylammonumhydroxıd $\times 30 \mathrm{H}_{2} \mathrm{O} 6 \mathrm{~h}$ zum Ruckfluß erhıtzt Dann wird 1 Vak vorsichtg eingeengt, 
$\mathrm{H}_{2} \mathrm{O}$ zugegeben und mit $1 \mathrm{~N}-\mathrm{HCl}$ angesüuert. Durch Ausschütteln mit $\mathrm{Et}_{2} \mathrm{O}$ (3x) und Abdampfen (1.Vak.) nach Trocknen uber $\mathrm{MgSO}_{4}$ - wird (S)-Phenylmichsăure (S)-14 erhalten. Für die Enanuomerenreınheitsbestummungen wird das Produkt noch durch Suure- Base-Extraktion gereinigt (1. 1N-NaOH zusetzen 2. mit $\mathrm{Et}_{2} \mathrm{O}$ schutteln (3x) 3. Ansăuern ( $\left.\mathrm{HCl}\right)$ 4. mit $\mathrm{Et}_{2} \mathrm{O}$ extrahıeren (3x), Trocknen (MgSO 4$)$, Eunengen). Zur Isolierung des Aminoalkohols wird die salzsaure Phase mit festem KOH alkalisch gestellt und sodann mit Pentan extrahrert (5x). Die vereinigten Pentanphasen werden getrocknet $\left(\mathrm{MgSO}_{4}\right)$ und eingeengt.

b) Wenn angegeben, wird die alkalische Phase zusätzlich noch mit $\mathrm{Et}_{2} \mathrm{O}(3 \mathrm{x})$ extrahiert und das dabei nach Trocknen mit $\mathrm{MgSO}_{4}$ und nach Einengen erhaltene Produkt sc (MeOH/NEtMe $2=9 / 1$ ) gereingt.

(R)-3,3-Dimethyl-1-I(R)-pyrroludin-2-yll-2-butanol ((R,R)-12a)

Nach AAV 4a aus $200 \mathrm{mg}(0.496 \mathrm{mmol})(R, R)-3 \mathrm{a}($ de $>98 \%)$ mit $1.69 \mathrm{~g}(2,75 \mathrm{mmol})$ TBAHx30 $\mathrm{H}_{2} \mathrm{O}$. (S)-14. $58 \mathrm{mg}(71 \%)$. $(R, R)-12 \mathrm{a}$ Farbluse Kristalle, Schinp. $62^{\circ} \mathrm{C}$, $[\alpha]_{546}=+29.3^{\circ},[\alpha]_{578}=+24.7^{\circ}\left(\mathrm{c}=0.93, \mathrm{CHCl}_{3}\right)$, Ausb $60 \mathrm{mg}(71 \%) .-\mathrm{C}_{10} \mathrm{H}_{21} \mathrm{NO}$ (171 3) Ber. C $701 \mathrm{H} 12.36 \mathrm{~N} 8.2 \mathrm{Gef}$. C $702 \mathrm{H} 1245 \mathrm{~N} 8.0$ Mol.- Masse 171 (ms) -IR $\left(\mathrm{CCl}_{4}\right)^{\circ} 3250 \mathrm{~cm}^{-1}-400 \mathrm{MHz}-{ }^{1} \mathrm{H}-\mathrm{NMR}\left(\mathrm{CDCl}_{3}\right): 087$ (s, 9H, $\mathrm{Bu}$ ), 1.16 (ddd, $\left.\mathrm{J}=106 / 116 / 141 \mathrm{~Hz}, 1 \mathrm{H}, \mathrm{CH}_{2} \mathrm{COH}\right), 133-1.41\left(\mathrm{~m}, 1 \mathrm{H}, \mathrm{NCH}_{2} \mathrm{CH}_{2} \mathrm{CH}_{2}\right), 147$ (ddd, $\mathrm{J}=1.5 / 31 / 141 \mathrm{~Hz}, 1 \mathrm{H}$, $\left.\mathrm{CH}_{2} \mathrm{COH}\right), 153-164\left(\mathrm{~m}, 1 \mathrm{H}, \mathrm{NCH}_{2} \mathrm{CH}_{2} \mathrm{CH}_{2}\right), 1.75-1.84\left(\mathrm{~m}, 1 \mathrm{H}, \mathrm{NCH}_{2} \mathrm{CH}_{2} \mathrm{CH}_{2}\right), 1.87-1.96(\mathrm{~m}, 1 \mathrm{H}$, $\mathrm{NCH}_{2} \mathrm{CH}_{2} \mathrm{CH}_{2}$ ), 279 (dt, J=11 $7 / 7 \mathrm{GH}, 1 \mathrm{H}, \mathrm{NCH}_{2}$ ), 2.94 (ddd, J=4.4/7.7/11.7Hz, $1 \overline{\mathrm{H}}, \mathrm{NCH}_{2}$ ), $331-335$ (m, $1 \mathrm{H}, \mathrm{NCH}), 338$ (dd, J=1 5/10 GHz, 1H, CHOH), 3.5-4.1 (s, breit, 1H), OH oder NH nicht lokalisiert.

\section{(S)-3,3-Dimethyl-1-[(R)-pyrrolidin-2-yll-2-butanol ( $R, S)-13 a)$}

Nach AAV 4a aus $200 \mathrm{mg}(0496 \mathrm{mmol})(R, S)-4 \mathrm{a}$ (de $>98 \%) \mathrm{mit} 169 \mathrm{~g}(275 \mathrm{mmol}) \mathrm{TBAHx} 30 \mathrm{H}_{2} \mathrm{O}$ Farbloser, wachsartiger Feststoff, Schmp $29-33^{\circ} \mathrm{C},[\alpha]_{546}=+6^{\circ},[\alpha]_{578}=+8^{\circ}\left(\mathrm{c}=0.75, \mathrm{CHCl}_{3}\right)$, Ausb $45 \mathrm{mg}$ (51\%) $-\mathrm{C}_{10} \mathrm{H}_{21} \mathrm{NO}(171$ 3) Ber. C $70.1 \mathrm{H} 12.36 \mathrm{~N} 8.2$ Gef. C 70.4 H 12.17 N 81 Mol.- Masse 171 (ms) -IR $\left(\mathrm{CCl}_{4}\right) 3250 \mathrm{~cm}^{-1}-400 \mathrm{MHz}-{ }_{\mathrm{H}} \mathrm{H}-\mathrm{NMR}\left(\mathrm{CDCl}_{3}\right) .0 .89(\mathrm{~s}, 9 \mathrm{H},-\mathrm{Bu}), 153$ (ddd, J=2 0/4 2/14.3Hz, $1 \mathrm{H}$, $\mathrm{CH}_{2} \mathrm{COH}$ ), 164 (ddd, $\mathrm{J}=41 / 10.7 / 14.3 \mathrm{~Hz}, 1 \mathrm{H}, \mathrm{CH}_{2} \mathrm{COH}$ ), $1.64-191$ (m, $4 \mathrm{H}, \mathrm{NCH}_{2} \mathrm{CH}_{2} \mathrm{CH}_{2}$ ), 286 (dt, $\mathrm{J}=101 / 73 \mathrm{~Hz}, 1 \mathrm{H}, \mathrm{NCH}_{2}$ ), 300 (ddd, $\left.\mathrm{J}=5.4 / 68 / 10 \mathrm{lHz}, 1 \mathrm{H}, \mathrm{NCH}_{2}\right), 350-356(\mathrm{~m}, 1 \mathrm{H}, \mathrm{NCH}$ ), 357 (dd, $\mathrm{J}=2$ 0/10 7Hz, 1H, CHOH), OH oder NH nicht lokalisiert

(R)-1-Phenyl-2- $((R)-2$-pyrrolidinyllethanol $((R, R)-12 b)$

Nach AAV $4 \mathrm{a}+\mathrm{b}$ aus $600 \mathrm{mg}(1.42 \mathrm{mmol})(R, R)-3 \mathrm{~b}$ (de $>98 \%)$ mit $6 \mathrm{~g}(972 \mathrm{mmol})$ TBAHx30 $\mathrm{H}_{2} \mathrm{O} 6 \mathrm{~h}$ (S)-14 $174 \mathrm{mg}(74 \%)$. ( $R, R)-12 \mathrm{~b}$ Farblose Kristalle, Schmp. $56^{\circ} \mathrm{C},[\alpha]_{546}=+405^{\circ},[\alpha]_{578}=+368^{\circ}(\mathrm{c}=093$, $\mathrm{CH}_{3} \mathrm{OH}$ ), Ausb $187 \mathrm{mg}(69 \%)-\mathrm{C}_{12} \mathrm{H}_{17} \mathrm{NO}(1913) \mathrm{Ber} \mathrm{C} 75.4 \mathrm{H} 8.96 \mathrm{~N} 73 \mathrm{Gef} \mathrm{C} 756 \mathrm{H} 897 \mathrm{~N} 71$ Mol.-Masse $191(\mathrm{~ms})$-IR $\left(\mathrm{CCl}_{4}\right) 3200 \mathrm{~cm}^{-1} .400 \mathrm{MHz}^{-1} \mathrm{H}-\mathrm{NMR}\left(\mathrm{CDCl}_{3}\right) \cdot 139$ (dddd, J=4.4/6.7/8.3/12 5Hz, $1 \mathrm{H}, \mathrm{NCH}_{2} \mathrm{CH}_{2} \mathrm{CH}_{2}$ ), 154 (ddd, J=10.6/11 4/14 3Hz, 1H, $\mathrm{CH}_{2} \mathrm{COH}$ ), 1.59-1.66 (m, 1H, $\left.\mathrm{NCH}_{2} \mathrm{CH}_{2} \mathrm{CH}_{2}\right), 1.67$ (ddd, $\left.\mathrm{J}=22 / 3.4 / 143 \mathrm{~Hz}, 1 \mathrm{H}, \mathrm{CH}_{2} \mathrm{COH}\right), 179-185\left(\mathrm{~m}, 1 \mathrm{H}, \mathrm{NCH}_{2} \mathrm{CH}_{2} \mathrm{CH}_{2}\right), 190-198(\mathrm{~m}, 1 \mathrm{H}$, $\mathrm{NCH}_{2} \mathrm{CH}_{2} \mathrm{CH}_{2}$ ), 287 (dt, J=11 $77.7 \mathrm{~Hz}, 1 \mathrm{H}, \mathrm{NCH}_{2}$ ), 301 (ddd, $\mathrm{J}=48 / 81 / 117 \mathrm{~Hz}, 1 \mathrm{H}, \mathrm{NCH}_{2}$ ), 358 (dddd, $\mathrm{J}=34 / 4 \overline{2 / 8} 1 / 11.4 \mathrm{~Hz}, 1 \mathrm{H}, \mathrm{NCH}), 32-45$ (s, brett, 1H), 488 (dd, J=2 2/10 6Hz, 1H, CHOH), $72-739$ (m, 5H, $\mathrm{Ph}), \mathrm{OH}$ oder NH micht lokalisiert.

(S)-1-Phenyl-2-I(R)-2-pyrroludinyllethanol (R,S)-13b)

Nach AAV $4 \mathrm{a}+\mathrm{b}$ aus $600 \mathrm{mg}(1.42 \mathrm{mmol})(R, S)-4 \mathrm{~b}$ (de $>98 \%) \mathrm{mit} 6 \mathrm{~g}(972 \mathrm{mmol})$ TBAHx $30 \mathrm{H}_{2} \mathrm{O}$. Farblose Kristalle, Schmp 103-104. $4^{\circ},[\alpha]_{546}=-16.5^{\circ},[\alpha]_{578}=-12^{\circ}\left(\mathrm{c}=0.665, \mathrm{CH}_{3} \mathrm{OH}\right)$, Ausb. $138 \mathrm{mg}$ (51\%) $-\mathrm{C}_{12} \mathrm{H}_{17} \mathrm{NO}$ (191.3) Ber C 75.4 H $896 \mathrm{~N} 73 \mathrm{Gef}$. C $756 \mathrm{H} 878 \mathrm{~N} 72 \mathrm{Mol}$-Masse 191 (ms) -IR $\left(\mathrm{CCl}_{4}\right) 3200 \mathrm{~cm}^{-1}-400 \mathrm{MHz}-{ }^{1} \mathrm{H}-\mathrm{NMR}\left(\mathrm{CDCl}_{3}\right) 151-159\left(\mathrm{~m}, 1 \mathrm{H}, \mathrm{NCH}_{2} \mathrm{CH}_{2} \mathrm{CH}_{2}\right), 160-169(\mathrm{~m}, 1 \mathrm{H}$, $\left.\mathrm{NCH}_{2} \mathrm{CH}_{2} \mathrm{CH}_{2}\right), 178-188\left(\mathrm{~m}, 4 \mathrm{H}, \mathrm{CH}_{2} \mathrm{COH}, \mathrm{NCH}_{2} \mathrm{CH}_{2} \mathrm{CH}_{2}\right), 292$ (ddd, J=5.9/7/11 Hz, 1H, NCH ), 297 $\left(\mathrm{dt}, \mathrm{J}=11 / 68 \mathrm{~Hz}, 1 \mathrm{H}, \mathrm{NCH}_{2}\right), 335(\mathrm{dq}, \mathrm{J}=44 / 7 \mathrm{~Hz}, 1 \mathrm{H}, \mathrm{NCH}), 5.04(\mathrm{dd}, \mathrm{J}=42 / 62 \mathrm{~Hz}, 1 \mathrm{H}, \mathrm{CHOH}), 72-74(\mathrm{~m}$, $5 \mathrm{H}, \mathrm{Ph}), \mathrm{OH}$ und $\mathrm{NH}$ nicht lokalisiert

(S)-1-Phenyl-2-I(S)-2-pyrrolidınyllethanol ((S,S)-12b)

Nach AAV $4 \mathrm{a}+\mathrm{b}$ aus $600 \mathrm{mg}$ (1 $42 \mathrm{mmol})(S, S)-5 \mathrm{~b}(\mathrm{de}>98 \%) \mathrm{mit} 6 \mathrm{~g}(972 \mathrm{mmol})$ TBAHx $30 \mathrm{H}_{2} \mathrm{O}$ Farblose Kristalle, Schmp $56^{\circ} \mathrm{C},[\alpha]_{546}=-411^{\circ},[\alpha]_{578}=-361^{\circ}\left(\mathrm{c}=076, \mathrm{CH}_{3} \mathrm{OH}\right)$, Ausb $149 \mathrm{mg}(55 \%)-\mathrm{C}_{12} \mathrm{H}_{17} \mathrm{NO}$ (1913) Ber C 75 4 H $896 \mathrm{~N} 73 \mathrm{Gef} \mathrm{C}^{\prime} 75 \mathrm{H} 873 \mathrm{~N} 70 \mathrm{Mol}$ - Masse 191 (ms) -IR und $400 \mathrm{MHz}$ - $\mathrm{H}-\mathrm{NMR}$ $\left(\mathrm{CDCl}_{3}\right)$ wie be1 $(R, R)-12 \mathrm{~b}$ beschrieben

(R)-1-Phenyl-2-I(S)-2-pyrrolidinyllethanol $((S, R)-13 \mathrm{~b})$

Aus $301 \mathrm{mg}(071 \mathrm{mmol})(S, R)-6 \mathrm{~b}$ (de $>98 \%) \mathrm{mit} 3 \mathrm{~g}$ (4 $86 \mathrm{mmol})$ TBAH x $30 \mathrm{H}_{2} \mathrm{O}$ Farblose Knstalle, Schmp $103-104^{\circ} \mathrm{C},[\alpha]_{546}=+16^{\circ},[\alpha]_{578}=+118^{\circ}\left(\mathrm{c}=0.58, \mathrm{CH}_{3} \mathrm{OH}\right)$, Ausb. $73 \mathrm{mg}(54 \%) .-\mathrm{C}_{12} \mathrm{H}_{17} \mathrm{NO}$ (1913) Ber C 754 H 896 N 73 Gef C 752 H 903 N 72 Mol - Masse 191 (ms) -IR und 400 MHz- H-NMR $\left(\mathrm{CDCl}_{3}\right)$ wie bei $(R, S)$-13b beschneben

(R)-1-(4-Chlorphenyl)-2-I(R)-2-pyrrolidinyllethanol $((R, R)-12 \mathrm{c})$

Nach AAV $4 \mathrm{a}+\mathrm{b}$ aus $896 \mathrm{mg}(176 \mathrm{mmol})(R, R)-3 \mathrm{c}(\mathrm{de}>98 \%) \mathrm{mt} 7.4 \mathrm{~g}(120 \mathrm{mmol}) \mathrm{TBAHx} 30 \mathrm{H}_{2} \mathrm{O}$ 
Abweichend davon wurde mit verd. $\mathrm{KOH}$ alkalisiert, der Aminoalkohol fiel dabei in der Wasserphase aus Farblose Knstalle, Schmp $113^{\circ} \mathrm{C},[\alpha]_{546}=+353^{\circ},[\alpha]_{578^{2}}=+31.5^{\circ}\left(\mathrm{c}=0.605, \mathrm{CH}_{3} \mathrm{OH}\right)$, Ausb $306 \mathrm{mg}$ (77\%). $-\mathrm{C}_{12} \mathrm{H}_{16} \mathrm{ClNO}$ (225 7) Ber. C 639 H 7.15 N 6.2 Gef. C 63.8 H 7.08 N 6.3 Mol.-Masse 225 (ms) -IR 3300,3080 (breit) $\mathrm{cm}^{-1}-400 \mathrm{MHz}-{ }^{-} \mathrm{H}-\mathrm{NMR}\left(\mathrm{CDCl}_{3}\right): 134-142$ (m, 1H, $\mathrm{NCH}_{2} \mathrm{CH}_{2} \mathrm{CH}_{2}$ ), 146 (ddd, $\mathrm{J}=106 / 114 / 14.2 \mathrm{~Hz}, 1 \mathrm{H}, \mathrm{CH}_{2} \mathrm{COH}$ ), $157-167$ (m, 1H, $\left.\mathrm{NCH}_{2} \mathrm{CH}_{2} \mathrm{CH}_{2}\right), 1.62$ (ddd, J=1.8/3.1/14 2Hz, $1 \mathrm{H}$, $\mathrm{CH}_{2} \mathrm{COH}$ ), $179-1.85\left(\mathrm{~m}, 1 \overline{\mathrm{H}}, \mathrm{NCH}_{2} \mathrm{CH}_{2} \mathrm{CH}_{2}\right), 1.87-1.97\left(\mathrm{~m}, 1 \mathrm{H}, \mathrm{NCH}_{2} \mathrm{CH}_{2} \mathrm{CH}_{2}\right), 2.86(\mathrm{dt}, \mathrm{J}=12 / 76 \mathrm{~Hz}, 1 \mathrm{H}$, $\mathrm{NCH}_{2}$ ), 302 (ddd, J=4 2/7 9/12Hz, $1 \mathrm{H}, \mathrm{NCH}_{2}$ ), 3.54-3.61 (m, $1 \mathrm{H}, \mathrm{NCH}$ ), 485 (dd, J=1 8/10 6Hz, $1 \mathrm{H}$, $\mathrm{CHOH}), 7$ 22-7.32 (m, 2H, $\left.\mathrm{C}_{6} \mathrm{H}_{4}\right), \mathrm{OH}$ und $\mathrm{NH}$ nicht lokalisiert.

(S)-1-(4-Chlorphenyl)-2-I(R)-2-pyrroludunyllethanol ( $(R, S)-13 \mathrm{c})$

Nach AAV 4a+b aus $950 \mathrm{mg}(208 \mathrm{mmol})(R, S)-4 \mathrm{c}$ (de $>98 \%)$ mit $8.74 \mathrm{~g}(142 \mathrm{mmol})$ TBAHx30 $\mathrm{H}_{2} \mathrm{O}$ Farblose Knstalle, Schmp. $146^{\circ} \mathrm{C},[\alpha]_{546}=-13.6^{\circ},[\alpha]_{578}=-102^{\circ}\left(\mathrm{c}=056, \mathrm{CH}_{3} \mathrm{OH}\right)$, Ausb. $355 \mathrm{mg}(76 \%)$ $-\mathrm{C}_{12} \mathrm{H}_{16} \mathrm{ClNO}(225.7)$ Ber C $63.9 \mathrm{H} 7.15 \mathrm{~N} 62 \mathrm{Gef} \mathrm{C} 639 \mathrm{H} 715 \mathrm{~N} 62 \mathrm{Mol}$-Masse 225 (ms) - IR 3260, 3120 (brett) $\mathrm{cm}^{-1} .400 \mathrm{MHz}^{1} \mathrm{H}-\mathrm{NMR}\left(\mathrm{CDCl}_{3}\right), 1.52-1.61\left(\mathrm{~m}, 1 \mathrm{H}, \mathrm{NCH}_{2} \mathrm{CH}_{2} \mathrm{CH}_{2}\right), 163-171(\mathrm{~m}, 1 \mathrm{H}$, $\left.\mathrm{NCH}_{2} \mathrm{CH}_{2} \mathrm{CH}_{2}\right), 1.76-1.89\left(\mathrm{~m}, 4 \mathrm{H}, \mathrm{CH}_{2} \mathrm{COH}, \mathrm{NCH}_{2} \mathrm{CH}_{2} \mathrm{CH}_{2}\right), 2.89-299(\mathrm{~m}, 2 \mathrm{H}, \overline{\mathrm{NCH}}$ ), $342(\mathrm{dq}, \mathrm{J}=49)$ $64 \mathrm{~Hz}, \overline{1 H}, \overline{\mathrm{NCH}}), 502$ (dd, J=2.9/7.8Hz, $1 \mathrm{H}, \mathrm{CHOH}), 7 \overline{24-736}\left(\mathrm{~m}, 4 \mathrm{H}, \mathrm{C}_{6} \mathrm{H}_{4}\right), \mathrm{OH}$ und $\mathrm{NH}$ nicht lokalisiert

(S)-1-(4-Chlorphenyl)-2-I(S)-2-pyrroludinyllethanol ((S,S)-12c)

Nach AAV $4 \mathrm{a}+\mathrm{b}$ aus $570 \mathrm{mg}(1.25 \mathrm{mmol})(S, S)-5 \mathrm{c}$ (de $>98 \%) \mathrm{mit} 53 \mathrm{~g}(86 \mathrm{mmol})$ TBAHx $30 \mathrm{H}_{2} \mathrm{O}$ Farblose Kristalle, Schmp $113^{\circ} \mathrm{C},[\alpha]_{546}=-38.1^{\circ},[\alpha]_{578}=-343^{\circ}\left(\mathrm{c}=0525, \mathrm{CH}_{3} \mathrm{OH}\right)$, Ausb $171 \mathrm{mg}(61 \%)$ $-\mathrm{C}_{12} \mathrm{H}_{16} \mathrm{CINO}(2257)$ Ber C $63.9 \mathrm{H} 715 \mathrm{~N} 62 \mathrm{Gef} \mathrm{C} 639 \mathrm{H} 711 \mathrm{~N} 61 \mathrm{Mol}$ - Masse 225 (ms) -IR und 400 $\mathrm{MHz}-\mathrm{H}-\mathrm{NMR}\left(\mathrm{CDCl}_{3}\right)$ wie be1 $(R, R)-12 \mathrm{c}$ beschrieben

(R)-1-(4-Chlorphenyl)-2-I(S)-2-pyrroludinyllethanol ((S,R)-13c)

Nach AAV 4a+b aus $300 \mathrm{mg}(066 \mathrm{mmol})(S, R)-6 \mathrm{c}(\mathrm{de}>98 \%) \mathrm{mit} 28 \mathrm{~g}(45 \mathrm{mmol})$ TBAHx30 $\mathrm{H}_{2} \mathrm{O}(S)-14$ $80 \mathrm{mg}(73 \%)(S, R)-13 \mathrm{c}$ Farblose Kristalle, Schmp $147^{\circ} \mathrm{C},[\alpha]_{546}=+123^{\circ},[\alpha]_{578}=+93^{\circ}\left(\mathrm{c}=045, \mathrm{CH}_{3} \mathrm{OH}\right)$, Ausb $113 \mathrm{mg} \mathrm{(77 \% )}-\mathrm{C}_{12} \mathrm{H}_{16} \mathrm{ClNO}(2257)$ Ber C $639 \mathrm{H} 715 \mathrm{~N} 62 \mathrm{Gef} \mathrm{C} 637 \mathrm{H} 714 \mathrm{~N} 62$ Mol -Masse $225(\mathrm{~ms})$-IR und $400 \mathrm{MHz}-\mathrm{H}-\mathrm{NMR}\left(\mathrm{CDCl}_{3}\right)$ wie bel $(R, S)$-13c beschrieben.

(R)-1-(4-Methoxyphenyl)-2-[(R)-2-pyrrolidinyllethanol ((R,R)-12d)

Nach AAV $4 \mathrm{a}+\mathrm{b}$ aus $900 \mathrm{mg}(199 \mathrm{mmol})(R, R)$-3d (de $>98 \%) \mathrm{mit} 84 \mathrm{~g}(136 \mathrm{mmol}) \mathrm{TBAHx} 30 \mathrm{H}_{2} \mathrm{O}$, Reaktionsdauer $55 \mathrm{~h}$ Farblose Knstalle, Schmp $60-62^{\circ} \mathrm{C},[\alpha]_{546}=+381^{\circ},[\alpha]_{578}=+332^{\circ}$ (c=0815, $\mathrm{CH}_{3} \mathrm{OH}$ ), Ausb $321 \mathrm{mg}(74 \%)-\mathrm{C}_{13} \mathrm{H}_{19} \mathrm{NO}_{2}$ (221 3) Ber C $706 \mathrm{H} 865 \mathrm{~N} 63 \mathrm{Gef} \mathrm{C} 705 \mathrm{H} 889 \mathrm{~N} 65$ Mol -Masse 221 (ms).-IR. 3300, 3140 (brett) $\mathrm{cm}^{-1} .-400 \mathrm{MHz}^{-1} \mathrm{H}-\mathrm{NMR}\left(\mathrm{CDCl}_{3}\right): 133-142$ (m, $1 \mathrm{H}$, $\mathrm{NCH}_{2} \mathrm{CH}_{2} \mathrm{CH}_{2}$ ), 152 (ddd, $\mathrm{J}=10.3 / 112 / 143 \mathrm{~Hz}, 1 \mathrm{H}, \mathrm{CH}_{2} \mathrm{COH}$ ), $159-167\left(\mathrm{~m}, 1 \mathrm{H}, \mathrm{NCH}_{2} \mathrm{CH}_{2} \mathrm{CH}_{2}\right.$ ), 164 (ddd, $\left.\mathrm{J=2} 2 / 33 / 143 \mathrm{~Hz}, 1 \mathrm{H}, \mathrm{CH}_{2} \mathrm{COH}\right), 177-186\left(\mathrm{~m}, 1 \mathrm{H}, \mathrm{NCH}_{2} \mathrm{CH}_{2} \mathrm{CH}_{2}\right), 187-197 \mathrm{~m}, 1 \mathrm{H}$, $\mathrm{NCH}_{2} \mathrm{CH}_{2} \mathrm{CH}_{2}$ ), 287 (dt, J=11 7/7.6Hz, $1 \mathrm{H}, \mathrm{NCH}_{2}$ ), 300 (ddd, J=4 8/8 1/11 7Hz, 1H, $\mathrm{NCH}_{2}$ ), $351-358$ (m, $1 \mathrm{H}, \mathrm{NCH}$ ), 380 (s, 3H, OMe), 483 (dd, J=2 2/10.3Hz, $1 \mathrm{H}, \mathrm{CHOH}$ ), 687 (d, J=8 8Hz, 2H, C $6 \mathrm{H}_{4}$ ), 730 (d, $\left.\mathrm{J}=8 \mathrm{HHz}, 2 \mathrm{H}, \mathrm{C}_{6} \mathrm{H}_{4}\right)$, OH und $\mathrm{NH}$ nicht lokalisiert

\section{(S)-1-(4-Methoxyphenyl)-2-[(R)-2-pyrrolidinyllethanol ( $R, S)-13 \mathrm{~d})$}

Nach AAV $4 \mathrm{a}+\mathrm{b}$ aus $915 \mathrm{mg}(202 \mathrm{mmol})(R, S)-4 \mathrm{~d}(\mathrm{de}>98 \%) \mathrm{mit} 84 \mathrm{~g}(136 \mathrm{mmol})$ TBAHx30 $\mathrm{H}_{2} \mathrm{O}(S)-14$

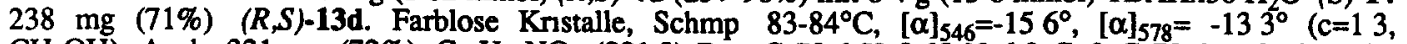
$\mathrm{CH}_{3} \mathrm{OH}$ ), Ausb $321 \mathrm{mg}(72 \%)-\mathrm{C}_{13} \mathrm{H}_{19} \mathrm{NO}_{2}(2213)$ Ber C $706 \mathrm{H} 865 \mathrm{~N} 63 \mathrm{Gef} \mathrm{C} 706 \mathrm{H} 870 \mathrm{~N} 64$ Mol -Masse 221 (ms) - IR 3250 , 3140 (breit) $\mathrm{cm}^{-1}-400-\mathrm{MHz}^{1} \mathrm{H}_{-\mathrm{NMR}}\left(\mathrm{CDCl}_{3}\right.$ ). $157-161$ (m, $1 \mathrm{H}$, $\mathrm{NCH}_{2} \mathrm{CH}_{2} \mathrm{CH}_{2}$ ), 1 64-1.71 (m, 1H, $\mathrm{NCH}_{2} \mathrm{CH}_{2} \mathrm{CH}_{2}$ ), 1 79-1 90 (m, 4H, $\mathrm{CH}_{2} \mathrm{COH}, \mathrm{NCH}_{2} \mathrm{CH}_{2} \mathrm{CH}_{2}$ ), 291 (ddd, $\mathrm{J}=55 / 77 / 12 \mathrm{~Hz}, 1 \mathrm{H}, \mathrm{NCH}_{2}$ ), $295\left(\mathrm{dt}, \mathrm{J}=12 / 68 \mathrm{~Hz}, 1 \mathrm{H}, \mathrm{NCH}_{2}\right), 342$ (quint, J=6 $2 \mathrm{~Hz}, 1 \mathrm{H}, \overline{\mathrm{NCH}}$ ), 380 (s, $3 \mathrm{H}$, OMe), 499 (t, J=5 1Hz, 1H, CHOH), $688\left(\mathrm{~d}, \mathrm{~J}=88 \mathrm{~Hz}, 2 \mathrm{H}, \mathrm{C}_{6} \mathrm{H}_{4}\right), 730$ (dd, J=8 8Hz, $2 \mathrm{H}, \mathrm{C}_{6} \mathrm{H}_{4}$ ), OH und $\mathrm{NH}$ nicht lokalisiert

(S)-1-(4-Methoxyphenyl)-2-I(S)-2-pyrrolidinyllethanol ((S,S)-12d)

Nach AAV $4 \mathrm{a}+\mathrm{b}$ aus $800 \mathrm{mg}(177 \mathrm{mmol})(S, S)-5 \mathrm{~d}$ (de $>98 \%) \mathrm{mit} 74 \mathrm{~g}(12.0 \mathrm{mmol})$ TBAHx30 $\mathrm{H}_{2} \mathrm{O}$ Farblose Kristalle, Schmp $60=62^{\circ} \mathrm{C},[\alpha]_{546}=-381^{\circ},[\alpha]_{578}=-312^{\circ}\left(\mathrm{c}=045, \mathrm{CH}_{3} \mathrm{OH}\right)$, Ausb $246 \mathrm{mg}$ $(63 \%)-\mathrm{C}_{13} \mathrm{H}_{19} \mathrm{NO}_{2}(2213)$ Ber C $706 \mathrm{H} 865 \mathrm{~N} 63 \mathrm{Gef} \mathrm{C} 707 \mathrm{H} 863 \mathrm{~N} 60 \mathrm{Mol}$ - Masse 191 (ms) -IR und $400 \mathrm{MHz}^{-} \mathrm{H}-\mathrm{NMR}\left(\mathrm{CDCl}_{3}\right)$ whe bei $(R, R)-12 \mathrm{~d}$ beschrieben

(R)-1-(4-Methoxyphenyl)-2-[(S)-2-pyrrolidinyllethanol ( $(S, R)-13 \mathrm{~d})$

Nach AAV $4 \mathrm{a}+\mathrm{b}$ aus $453 \mathrm{mg}(1 \mathrm{mmol})(S, R)-6 \mathrm{~d}(\mathrm{de}>98 \%)$ mit $42 \mathrm{~g}(78 \mathrm{mmol})$ TBAHx30 $\mathrm{H}_{2} \mathrm{O}$ Farblose Kristalle, Schmp $81-82^{\circ} \mathrm{C},[\alpha]_{546}=+133^{\circ},[\alpha]_{578}=+10.7^{\circ}\left(\mathrm{c}=045, \mathrm{CH}_{3} \mathrm{OH}\right)$, Ausb $141 \mathrm{mg}$ $(65 \%)-\mathrm{C}_{13} \mathrm{H}_{19} \mathrm{NO}_{2}$ (221.3) Ber. C $706 \mathrm{H} 865 \mathrm{~N} 63 \mathrm{Gef} \mathrm{C} 708 \mathrm{H} 859 \mathrm{~N} 61$ Mol.- Masse 191 (ms) -IR und $400 \mathrm{MHz}^{-1} \mathrm{H}-\mathrm{NMR}\left(\mathrm{CDCl}_{3}\right)$ we ber $(R, S)-13 \mathrm{~d}$ beschneben 
Enantiomerenreinheit von ( $S$ )-14

Die Derivatusierung von (S)-14 mit (S)-1-Naphthylethylamin und die Produltanalyse mit HPLC erfolgte nach dem in $\mathrm{Lit}^{25}$ angegebenen Verfahren. Die eingesetzte Menge an (S)-14 betrug jeweils $29 \mathrm{mg}(0.175 \mathrm{mmol})$ Die Proben stammten aus der Hydrolyse von:

a) $(R, R)-3 \mathrm{~b}$ b) $(R, S)-4 \mathrm{~d}$ c) $(S, R)-6 \mathrm{c}$.

Die Ergebnisse der HPLC-Analyse sind in Tab. 7 zusammengestellit

Danksagung Wir danken Herrn Prof. F. E1den für die großzügige Förderung und der Deutschen Forschungsgemeinschaft sowie den Fonds der Chemischen Industre für die finanzelle Unterstützung dieser Arbeit.

\section{LITERATUR}

1 a) Wang, Y -F, Izawa, T, Kobayash1, S.; Ohno, M. J Am Chem Soc 1982, 104, 6465 b) Hahn, H, Heitsch, H., Rathmann, R ; Zimmermann, Gesıne, Bormann, C, Zähner, H ; Könı, W.A Liebıgs Ann Chem 1987, 803

2 Southon, I W ; Buckıngham, J "Dictionary of Alkaloıds" Chapman and Hall, London, New York 1989

3 Eisstetten, K, Kley, H -P (Byk Gulden), Ger Offen. 2852088 (1979), C A 1979, 91, $91498 \mathrm{~m}$

4 Bross1, A, Besendorf, H, Purk, L.A, Rheiner, A.. "Isoquinoline Analgetıcs". In Analgetics, Medicinal Chemistry (G DeStevens, ed ), Bd. 5, S. 281, Academic Press, London 1965

5 Bonginı, A., Cardıllo, G, Orena, M ; Porz, G., Sandn, S Chem Lett 1988, 87.

6 Ito, S., Hurama, M. Heterocycles 1989, 28, 1229

7 a) Jäger, V ; BuB, V Liebigs Ann Chem 1980, 101 b) Jăger, V, BuB, V ; Schwab, W. Liebigs Ann Chem 1980, 122 c) Jager, V ; Schohe, R Tetrahedron 1984, 40, 2199 d) Wade, P A., Price, D.T Tetrahedron Lett 1989, 30, 1185

8 Mulzer, J Nachr Chem Tech Lab 1984, 32, 882 Liguon, A ; Ottana, R., Romeo, G., Sindona, G, Uccella, N Chem Ber 1989, 122, 2019. Wovkulich, P M ; Uskokovic, M.R.J Am Chem Soc 1981, 103, 3956 C1cch1, St., Got, A , Brand, A , Guarna, A.; De Sarlo, F Tetrahedron Lett 1990, 31, 3351. Tufanello, J J. Acc Chem Res 1979, 12, 396

9 a) Yamamoto, Y, Komatsu, T ; Maruyama, K Chem Commun 1985, 814. b) Narasaka, K, Ukaj1, Y, Yamazak, S Bull Chem Soc Jpn 1986, 59,525

10 Tramontn1, M Synthests 1982, 605. Barluenga, J , Olano, B ; Fustero, S J Org Chem 1985, 50 , 4052 Pill, R A, Russowsky, D, Dias, L C J Chem Soc Perkan Trans I 1990, 1213

11 Schöpf, C, Dummer, G, Wust, W, Rausch, R Liebigs Ann Chem 1959, 626, 134 Roessler, F, Ganzinger, D, Johne, S ; Schopp, E ; Hesse, M. Helv Chum Acta 1978, 61, 1200

12 Kober R, Papadopoulos, K, Miltz, W., Enders, D, Steglich, W Tetrahedron 1985, 41, 1693 Shono T , Matsumura, Y, Tsubata, K ; Uchida, K. J Org Chem 1986, 51, 2590 Irie, K, Aoe, K., Tanaka, T , Sauto, S Chem Commun 1985, 633 Renaud, P, Seebach, D. Helv Chum Acta 1986, 69, 1704

Sinclair, P.J , Zha1, D., Reibenspies, J, Williams, R M J Am Chem Soc 1986, 108, 1103 Wanner, K Th, Praschak, I Arch Pharm (Weinheim) 1990, 323, 335

Wanner, K Th ; Höfner, G Arch Pharm (Weinheim) im Druck Wanner, K Th., Kärtner, A Arch Pharm (Weinheim) 1987, 320, 1253

16 Benassı, R, Foll, U ; Schenett, L, Taddeı F Adv Heterocycl Chem 1987, 41, 75 . Verbindungen siehe Reetz, M T Angew Chem 1984, 96, 542

18 Fur Chelat-kontrollierte Reaktionen be1 1,4-Dicarbonylverbindungen siehe Kunz, T, Re1ßig, H U , Janowitz, A Chem Ber 1989, 122, 2165

19 Larcheveque, M; Lalande, J Chem Commun 1985, 83.

20 Boone, J R ; Ashby, E.C. Top Stereochem 1979, 11, 53

21 Yamamoto, Y, Maruyama, K J Am Chem Soc 1985, 107, 6411

22 Fur die Anwendung dieser Begniffe auf eine intramolekulare doppelte Stereodifferenzierung siehe 9 a)

23 Zum selben Ergebnis fur das Racemat von $12 \mathrm{~b}$ kamen unabhangig von uns Tirel, P -J , Vaultier, M , Carre, R Tetrahedron Lett 1989, 30, 1947

24 Als Erklăung für die ${ }^{1} \mathrm{H}-\mathrm{NMR}$-Daten eıner zu $13 \mathrm{~b}$ analogen Pipendınverbındung Hootele, $\mathrm{C}$, Halın, F , Thomas, S , Tourwe, D Tetrahedron 1989, 41, 5563

25 Wanner, K Th, Höfner, G Arch Pharm (Weinheim) 1989, 322, 99.

26 Wanner, K Th, Höfner, G. Arch Pharm (Weinherm) 1989, 322, 93

27 Hisao, N, Kojı, N, Tamio, V, Naokazu, S Bull Chem Soc Jpn 1975, 48, 553 